\title{
COHEN-MACAULAYNESS OF SEMI-INVARIANTS FOR TORI
}

\author{
MICHEL VAN DEN BERGH
}

\begin{abstract}
In this paper we give a new method, in terms of one-parameter subgroups, to study semi-invariants for algebraic tori. In some cases we obtain extensions to results in [7]. In other cases we obtain different proofs.
\end{abstract}

\section{INTRODUCTION}

Let $T$ be an algebraic torus over an algebraically closed field of characteristic zero and let $W$ be a finite-dimensional representation of $T$. Then $T$ acts on the polynomial ring $R=k[W]$ and by the Hochster-Roberts theorem [3], $k[W]^{T}$ is Cohen-Macaulay.

Let $\chi$ be some character of $T$. In [7,8] Stanley defines $R_{\chi}^{T}$ to be the sum of all one-dimensional $T$-representations having character $\chi$. It is clear that $R_{\chi}^{T}$ is an $R^{T}$-module. $R_{\chi}^{T}$ occurs naturally in the study of linear diophantine equations.

An interesting question is what is the depth of $R_{\chi}^{T}$, in particular when $R_{\chi}^{T}$ is Cohen-Macaulay. Stanley gives an answer to this question in terms of certain polyhedral complexes whose dimension is of the order of $\operatorname{dim} W$ [7].

If $G$ is an arbitrary algebraic group then in [9] we introduced a method to study $R_{\chi}^{G}\left(R_{\chi}^{G}\right.$ is defined similarly as $\left.R_{\chi}^{T}\right)$. If $G=T$ is a torus then this approach is different from Stanley's method.

In the present paper we apply our techniques to the torus case. We obtain a description of the local cohomology modules $H_{\left(R^{T}\right)^{+}}^{\cdot}\left(R^{T}\right)$ in terms of oneparameter subgroups of $T$ (3.4.2). In this way one can compute the depth of $R_{\chi}^{T}$. With our method we still need some spherical complexes, but these have dimension of the order of $\operatorname{dim} T$.

The methods used in this computation turn out to be a key ingredient in [10] where the same questions are studied for general reductive groups. There the situation is much more intractable, and it seems to be impossible to obtain an answer as complete as in the torus case.

Section 4 is devoted to some applications of our results. If $\operatorname{dim} T=1$, we recover [7, Corollary 3.4$]$. We also completely analyze the case $\operatorname{dim} T=2$ and we obtain a generalization [7, Corollary 3.4].

In $\S 4.2$ we show that $R_{\chi}^{T}$ is Cohen-Macaulay if the same holds for all oneparameter subgroups of $T$ (4.2.1).

Received by the editors June 23, 1989 and, in revised form, November 13, 1990.

1980 Mathematics Subject Classification (1985 Revision). Primary 13C15, 05 A15.

The author is supported by an NFWO grant. 
In $\S 4.3$ we give a different proof of [7, Theorem 3.2].

In $\S 4.4$ we obtain (under some mild a priori hypothesis) necessary and sufficient conditions under which there exist only a finite number of $\chi$ for which $R_{\chi}^{T}$ is Cohen-Macaulay. It turns out that this is almost always the case.

In $\S 4.5$ we compute an explicit example and we obtain interesting counterexamples to the converses of [7, Theorem 3.2] and 4.2.1.

In $\S 4.6$ we give necessary and sufficient conditions for reciprocity [7] in terms of one-parameter subgroups of $T$. In particular we obtain that if $\operatorname{dim} T \leq 3$ and if reciprocity holds then $R_{\chi}^{T}$ is Cohen-Macaulay.

\section{NOTATION AND CONVENTIONS}

In this paper $k$ always denotes an algebraically closed field of characteristic zero.

If $T$ is a torus over $k$ then $X(T)$ resp. $Y(T)$ will be the character group and the group of one-parameter subgroups of $T$. These are both free abelian groups and therefore the group laws will be written additively. We define $X(T)_{\mathbb{R}}$ as $\mathbb{R} \otimes_{\mathbf{Z}} X(T)$ and $Y(T)_{\mathbf{R}}$ as $\mathbb{R} \otimes_{\mathbb{Z}} Y(T)$. There is a natural pairing $Y(T) \times X(T) \rightarrow$ $X\left(G_{m}\right)=\mathbb{Z}$ given by composition. This pairing will be denoted by $\langle$,$\rangle . We$ will extend this pairing to $Y(T)_{\mathbf{R}} \times X(T)_{\mathbb{R}}$.

We will also choose a positive definite quadratic form on $Y(T)_{\mathbb{R}}$. The corresponding norm will be denoted by \|\| . Then we define

$$
B(T)=\{\lambda \in Y(T) \mid\|\lambda\|<1\} \quad \text { and } S(T)=\{\lambda \in Y(T) \mid\|\lambda\|=1\} .
$$

Of course the results in this paper do not depend on the choice of \|\| .

Characters of $T$ will be identified with one-dimensional representations of $T$. Hence the notation $\chi_{1} \oplus \chi_{2}$ for $\chi_{1,2} \in X(T)$ stands for the two-dimensional representation of $T$ which is the direct sum of the one-dimensional representations determined by $\chi_{1}$ and $\chi_{2}$. This is not to be confused with $\chi_{1}+\chi_{2}$ which is just the sum of $\chi_{1}$ and $\chi_{2}$ in $X(T)$.

Let $d$ be some positive integer and assume that $V$ is a $\mathbb{Z}^{d}$-graded $k$-vector space. If $x$ is a homogeneous element in $V$ then $\operatorname{deg} x$ will be the degree of $x$. If $a=\left(a_{1}, \ldots, a_{d}\right) \in \mathbb{Z}^{d}$ then $V_{a}$ will be the homogeneous part of $V$ of degree $a$.

The Poincaré series of $V$ is defined as

$$
P(V, t)=\sum_{a \in \mathbb{Z}^{d}} \operatorname{dim}\left(V_{a}\right) t^{a},
$$

where $t=\left(t_{1}, \ldots, t_{d}\right)$ and $t^{a}=t_{1}^{a_{1}} \cdots t_{d}^{a_{d}}$. (1) is usually supposed to define an element of $t^{-\gamma} \mathbb{Z}\left[\left[t_{1}, \ldots, t_{d}\right]\right]$ for some $\gamma \in \mathbb{Z}^{d}$. However in some occasions (1) will define an element of $t^{\gamma} \mathbb{Z}\left[\left[t_{1}^{-1}, \ldots, t_{d}^{-1}\right]\right]$.

If $E$ is a vector space and $x_{1}, \ldots, x_{d} \in E$ is a set of points then by $\operatorname{span}\left\{\left(x_{i}\right)_{i=1, \ldots, d}\right\}$ we denote the subvector space of $E$ spanned by $\left(x_{i}\right)_{i=1, \ldots, d}$. By $\operatorname{pos}\left\{\left(x_{i}\right)_{i=1, \ldots, d}\right\}$ we mean the set of all positive linear combinations of $\left(x_{i}\right)_{i=1, \ldots, d}$. This is a polyhedral cone (see [1]).

If $A$ is a polyhedral cone or a spherical polyhedral set, then by the apex set, of $A$ (notation: apex $A$ ) we denote the set $A \cap-A$. If $A$ is a polyhedral cone then apex $A$ is a linear subspace of $A$.

In the sequel we will be mostly concerned with the following situation. An $s$ dimensional torus acts on a $d$-dimensional vector space $W$ with basis $\left\{w_{1}, \ldots\right.$, 
$w_{d}$ \} and corresponding weights $\alpha_{1}, \ldots, \alpha_{d}$. To simplify the notation we will put $E=X(T)_{\mathbb{R}}, E^{*}=Y(T)_{\mathbb{R}}, S=S(T), B=B(T)$.

Some other frequently used notations are $R=k[W], X=\operatorname{Spec} k[W]$, $h=\operatorname{dim} R^{T}=\operatorname{dim} X / T, \mathscr{W}=\{1, \ldots, d\}$ (the index set for the weights of $W), X^{u}$ is the unstable locus in $X, X_{\lambda}=\left\{x \in X \mid \lim _{t \rightarrow 0} \lambda(t) x=0\right\}$, $T_{\lambda}=\left\{i \in \mathscr{W} \mid\left\langle\lambda, \alpha_{i}\right\rangle<0\right\}, d_{\lambda}=d-\left|T_{\lambda}\right|, \lambda \sim \lambda^{\prime} \Leftrightarrow T_{\lambda}=T_{\lambda^{\prime}}, \Lambda=B / \sim$, $B_{\lambda}=\{\mu \in B \mid \mu \sim \lambda\}, S_{\lambda}=\{\mu \in S \mid \mu \sim \lambda\}, \Phi_{\lambda}=\overline{B_{\lambda}}-B_{\lambda}, \Phi_{\lambda}^{\prime}=\overline{S_{\lambda}}-S_{\lambda}, M$ is the subsemigroup of $X(T)$ generated by $\left(\alpha_{i}\right)_{i=1, \ldots, d}, E_{\lambda}=\operatorname{span}\left\{\left(\alpha_{i}\right)_{i \in T_{\lambda}}\right\}$, $A_{\lambda}=\operatorname{pos}\left\{\left(\alpha_{i}\right)_{i \in T_{\lambda}}\right\}, B_{\lambda}=\operatorname{pos}\left\{\left(\alpha_{i}\right)_{i \in T_{\lambda}},\left(-\alpha_{i}\right)_{i \notin T_{\lambda}}\right\}, u_{\lambda}=\operatorname{dim} E_{\lambda}$.

\section{LOCAL COHOMOLOGY OF SEMI-INVARIANTS}

3.1 Generalities. Let $G$ be a reductive algebraic group over $k$ and let $W$ be a finite-dimensional representation of $G$. Define $R=k[W], d=\operatorname{dim} W$, $h=\operatorname{dim} R^{G}$ and let $\chi$ be an irreducible character of $G$.

In [8] Stanley defines $R_{\chi}^{G}$ as the sum of all irreducible representations of $G$ in $R$, having character $\chi$. Note that $R=\bigoplus_{\chi} R_{\chi}^{G}$ where $\chi$ runs over all irreducible characters of $G$. Furthermore it is easy to see that $R_{\chi}^{G}$ is finitely generated over $R^{G}$ and hence $\operatorname{dim} R_{\chi}^{G}=\operatorname{dim} R^{G}$ if $R_{\chi}^{G} \neq 0$. One is often interested in the depth of $R_{\chi}^{G}$ or, more specifically, in the question of when $R_{\chi}^{G}$ is Cohen-Macaulay. See e.g. [9].

The following elementary lemma was used in [9]. Define $I=R\left(R^{G}\right)^{+}$.

Lemma 3.1.1. $H_{\left(R^{G}\right)^{+}}^{\cdot}\left(R_{\chi}^{G}\right)=H_{I}(R)_{\chi}^{G}$ (with obvious notations).

Proof. Let $f_{1}, \ldots, f_{u}$ be a set of generators for $\left(R^{G}\right)^{+}$. Then the $\left(f_{i}\right)_{i}$ are obviously also $R$-generators for $I$. Let $K^{*}\left(R, f_{1}, \ldots, f_{u}\right)$ be the complex

$$
0 \rightarrow R \rightarrow \bigoplus_{i} R_{f_{i}} \rightarrow \bigoplus_{i, j ; i<j} R_{f_{i} f_{j}} \rightarrow \cdots \rightarrow R_{f_{1} \cdots f_{u}} \rightarrow 0
$$

with the standard boundary maps. Then $H_{I}^{\cdot}(R)_{\chi}^{G}=H^{\bullet}\left(K^{\bullet}\left(R, f_{1}, \ldots, f_{u}\right)\right)_{\chi}^{G}$. But, using the fact that $G$ is reductive, we deduce

$$
\begin{aligned}
H^{\bullet}\left(K^{\cdot}\left(R, f_{1}, \ldots, f_{u}\right)\right)_{\chi}^{G} & =H^{\cdot}\left(K^{\cdot}\left(R, f_{1}, \ldots, f_{u}\right)_{\chi}^{G}\right) \\
& =H^{\cdot}\left(K^{\cdot}\left(R_{\chi}^{G}, f_{1}, \ldots, f_{u}\right)\right) \\
& =H_{\left(R^{G}\right)^{+}}^{\cdot}\left(R_{\chi}^{G}\right),
\end{aligned}
$$

which is what we want.

Hence if $R_{\chi}^{G} \neq 0$ then $R_{\chi}^{G}$ is Cohen-Macaulay if and only if $H_{I}^{i}(R)_{\chi}^{G}=0$ for $i=0, \ldots, h-1$.

Assume now that $G=T$ is an $s$-dimensional torus. In that case $R_{\chi}^{T}=\{r \in$ $R \mid z . r=\chi(z) r$ for $z \in T\}$. Hence $R_{\chi}^{T}$ is an $R^{T}$-module of semi-invariants for the character $\chi$.

We may choose a basis $\left(w_{1}, \ldots, w_{d}\right)$ in $W$ such that the action of $T$ on $W$ is diagonal with respect to this basis; i.e., if $z \in T$ then $z . w_{i}=\alpha_{i}(z) w_{i}$ where the $\left(\alpha_{i}\right)_{i=1, \ldots, d} \in X(T)$ are the weights of $W$.

We will sometimes assume that $T$ acts faithfully on $W$. It is easy to see that this is equivalent with the following condition

$$
\operatorname{span}\left\{\left(\alpha_{i}\right)_{i=1, \ldots, d}\right\}=X(T)_{\mathbb{R}} .
$$


We can make $R$ into a $\mathbb{Z}^{d}$-graded ring by putting

$$
\operatorname{deg}\left(w_{i}\right)=(0, \ldots, 0,1,0, \ldots, 0)
$$

where the one occurs in the $i$ th position. This grading is obviously compatible with the $T$-action and hence $R^{T}$ and $R_{\chi}^{T}$ are also graded $k$-vector spaces.

It will be clear from Lemma 3.1.1 that our aim should be to describe $H_{I}^{*}(R)$. To this end we introduce some geometric notions.

Let $X=\operatorname{Spec} k[W]$. Then the radical of the ideal $I$ is the defining ideal of the $T$-unstable locus in $X$, which will be denoted by $X^{u}$; i.e.;

$$
X^{u}=\{x \in X \mid 0 \in \overline{T x}\} .
$$

Using this notation we may write $H_{I}^{*}(R)=H_{X^{u}}\left(X, \mathscr{O}_{X}\right)$.

$X^{u}$ may be described more conveniently using the Hilbert-Mumford criterion [6] which says that every point in $X^{u}$ is unstable for some one-parameter subgroup of $T$; i.e., if $\lambda \in Y(T)$ then one defines $X_{\lambda}=\left\{x \in X \mid \lim _{t \rightarrow 0} \lambda(t) x=\right.$ $0\}$ and one obtains

$$
X^{u}=\bigcup_{\lambda \in Y(T)} X_{\lambda} .
$$

The closed points of $X$ are in one-one correspondence with the elements of $W^{*}$. Let $w_{1}^{*}, \ldots, w_{d}^{*}$ be the dual basis of $W^{*}$. Then $\lambda$ acts on $W^{*}$ by $z \rightarrow$ $\operatorname{diag}\left(z^{-\left\langle\lambda, \alpha_{1}\right\rangle}, \ldots, z^{-\left\langle\lambda, \alpha_{d}\right\rangle}\right)$. Hence $X_{\lambda}$ is the linear subspace of $X$ spanned by the $w_{i}^{*}$ where $\left\langle\lambda, \alpha_{i}\right\rangle<0$.

Note that this description of $X_{\lambda}$ makes sense if $\lambda \in Y(T)_{\mathbb{R}}$. Hence we will also use the notation $X_{\lambda}$ for $\lambda \in Y(T)_{\mathbb{R}}$. However it is easy to see that there always exists a $\lambda^{\prime} \in Y(T)$ such that $X_{\lambda}=X_{\lambda^{\prime}}$.

To simplify the notation we will put $E=X(T)_{\mathbb{R}}, E^{*}=Y(T)_{\mathbb{R}}, S=S(T)$, and $B=B(T)$.

If $U \subset E^{*}$ then we define $X_{U}=\bigcup_{\lambda \in U} X_{\lambda}$. Using this notation, (3) may be rephrased as

$$
X^{u}=X_{Y(T)}=X_{E^{*}}=X_{\bar{B}} .
$$

Let $\mathscr{W}=\{1, \ldots, d\}$. We define $T_{\lambda}=\left\{i \in \mathscr{W} \mid\left\langle\lambda, \alpha_{i}\right\rangle<0\right\}$.

The following lemmas will be needed in the following sections.

Lemma 3.1.2. Assume that $U_{1,2}$ are closed convex subsets of $E^{*}$ such that $U_{1} \cup$ $U_{2}$ is convex. Then $X_{U_{1} \cap U_{2}}=X_{U_{1}} \cap X_{U_{2}}$.

Proof. It is clear that $X_{U_{1} \cap U_{2}} \subset X_{U_{1}} \cap X_{U_{2}}$. To prove the opposite inclusion, take $\lambda_{1} \in U_{1}, \lambda_{2} \in U_{2}$. We have to find $\lambda \in U_{1} \cap U_{2}$ such that $X_{\lambda_{1}} \cap X_{\lambda_{2}} \subset X_{\lambda}$ or equivalently $T_{\lambda_{1}} \cap T_{\lambda_{2}} \subset T_{\lambda}$. Using the hypothesis, we deduce that $\left[\lambda_{1}, \lambda_{2}\right] \cap$ $U_{1} \cap U_{2} \neq \varnothing$. Hence we may take $\lambda \in\left[\lambda_{1}, \lambda_{2}\right] \cap U_{1} \cap U_{2}$.

If $i \in T_{\lambda_{1}} \cap T_{\lambda_{2}}$ then $\left\langle\lambda_{1}, \alpha_{i}\right\rangle<0,\left\langle\lambda_{2}, \alpha_{i}\right\rangle<0$. But then it follows that also $\left\langle\lambda, \alpha_{i}\right\rangle<0$. Hence $i \in T_{\lambda}$.

Lemma 3.1.3. If $U \subset E^{*}$ then $X_{U}=X_{\bar{U}}$.

Proof. If $\mathscr{W}^{\prime} \subset \mathscr{W}$ then the condition $T_{\lambda} \supset \mathscr{W}^{\prime}$ is an open condition on $\lambda$. From this the result follows. 
3.2 A spectral sequence. We keep the same notation as before.

As we have seen, we have to be able to compute $H_{X_{u}}\left(X, \mathscr{O}_{X}\right)$. Furthermore $X^{u}=X_{\bar{B}}$. In this section we construct a spectral sequence which may be used to compute $H_{X_{U}}\left(X, \mathscr{O}_{X}\right)$ where $U$ is a bounded closed convex subset of $E^{*}$.

Let $\chi_{1}, \ldots, \chi_{m} \in E$ and let $\mathscr{P}$ be the set of all nonempty subsets of $U$ and $\partial U$ of the form

$$
\begin{array}{r}
\left\{\lambda \in U \mid\left\langle\lambda, \chi_{i}\right\rangle \geq 0 \text { for } i \in \mathscr{W}_{1},\left\langle\lambda, \chi_{i}\right\rangle=0 \text { for } i \in \mathscr{W}_{2},\right. \\
\left.\left\langle\lambda, \chi_{i}\right\rangle \leq 0 \text { for } i \in \mathscr{W}_{3}\right\}
\end{array}
$$

and

$$
\begin{array}{r}
\left\{\lambda \in \partial U \mid\left\langle\lambda, \chi_{i}\right\rangle \geq 0 \text { for } i \in \mathscr{W}_{1},\left\langle\lambda, \chi_{i}\right\rangle=0 \text { for } i \in \mathscr{W}_{2},\right. \\
\left.\left\langle\lambda, \chi_{i}\right\rangle \leq 0 \text { for } i \in \mathscr{W}_{3}\right\},
\end{array}
$$

where $\mathscr{W}=\mathscr{W}_{1} \cup \mathscr{W}_{2} \cup \mathscr{W}_{3}$ is an arbitrary decomposition of $\mathscr{W}$ as a disjoint union.

Furthermore $\mathscr{P}^{1}=\{\sigma \in \mathscr{P} \mid \sigma \subset \partial U\}$ and $\mathscr{P}^{0}=\mathscr{P} \backslash \mathscr{P}_{1}$.

If $\sigma, \sigma^{\prime} \in \mathscr{P}$ then we will say that $\sigma$ is a face of $\sigma^{\prime}$ if $\sigma \subset \sigma^{\prime} . \sigma$ is a facet of $\sigma^{\prime}$ if it is a face and $\operatorname{dim} \sigma=\operatorname{dim} \sigma^{\prime}-1$.

Let $\operatorname{dim} U=t$. We will assume that we have chosen $\left(\alpha_{\sigma, \sigma^{\prime}}\right)_{\sigma, \sigma^{\prime} \in \mathscr{P} 0} \in$ $\{-1,1,0\},\left(\beta_{\sigma}\right)_{\sigma \in \mathscr{P} 0, \operatorname{dim} \sigma=t} \in\{-1,+1\}$ with the following properties:

1. $\alpha_{\sigma^{\prime}, \sigma}=0$ unless $\sigma$ is a facet of $\sigma^{\prime}$. In that case $\alpha_{\sigma^{\prime}, \sigma}= \pm 1$.

2. If $\sigma, \sigma^{\prime \prime} \in \mathscr{P}^{0}, \operatorname{dim} \sigma^{\prime \prime}=\operatorname{dim} \sigma+2$, then

$$
\sum_{\sigma^{\prime} \in \mathscr{P} 0 ; \operatorname{dim} \sigma^{\prime}=\operatorname{dim} \sigma+1} \alpha_{\sigma^{\prime \prime}, \sigma^{\prime}} \alpha_{\sigma^{\prime}, \sigma}=0 .
$$

3. Let $\sigma \in \mathscr{P}^{0}, \operatorname{dim} \sigma=t-1$, then

$$
\beta_{\sigma_{1}^{\prime}} \alpha_{\sigma_{1}^{\prime}, \sigma}+\beta_{\sigma_{2}^{\prime}} \alpha_{\sigma_{2}^{\prime}, \sigma}=0
$$

where $\sigma_{1}^{\prime}, \sigma_{2}^{\prime}$ are the two elements of $\mathscr{P}^{0}$ having $\sigma$ as a facet.

As a final bit of notation we define for $U \subset V \subset E$ and for $\mathscr{F}$ a sheaf on $X$, the maps $i_{U, V}(\mathscr{F})$ as the natural homomorphisms $H_{X_{U}}^{\cdot}(X, \mathscr{F}) \rightarrow H_{X_{V}}^{\cdot}(X, \mathscr{F})$ associated to the inclusions $X_{U} \subset X_{V}$.

Theorem 3.2.1. With notation as above. Let $\mathscr{F}$ be a quasicoherent sheaf on $X$. Then there is a spectral sequence

$$
E_{p q}^{1}: \bigoplus_{\substack{\sigma \in \mathscr{P}^{0} \\ \operatorname{dim} \sigma=p}} H_{X_{\sigma}}^{q}(X, \mathscr{F}) \Rightarrow H_{X_{U}}^{p+q-t}(X, \mathscr{F}),
$$

where the boundary maps $d_{p q}$ are given by $\bigoplus_{\sigma^{\prime}} \sum_{\sigma} \alpha_{\sigma^{\prime}, \sigma} i_{\sigma, \sigma^{\prime}}(\mathscr{F})$. Here the sum runs over all pairs $\left(\sigma, \sigma^{\prime}\right)$ such that $\sigma$ is a maximal face of $\sigma^{\prime}$ and has dimension $p$.

Theorem 3.2.1 will be applied as follows. Assume that the $\chi_{1}, \ldots, \chi_{m}$ are chosen in such a way that the elements of $\mathscr{P}$ are the closed cells of a pseudomanifold structure with boundary on $U$. We may then choose a set of incidence numbers $\left(\alpha_{\sigma^{\prime}}, \sigma\right)_{\sigma^{\prime}, \sigma \in \mathscr{P}}$ as in [5, Theorem IV 7.2]. They obviously satisfy conditions 1 and 2.

Since $U$ is orientable, we may choose a coherent orientation on $U[5$, IV paragraph 8]. From this one deduces there exist $\left(\beta_{\sigma}\right)_{\sigma}$ satisfying condition 3. 
To prove Theorem 3.2.1 we need the following lemma.

Lemma 3.2.2. Assume that $\mathscr{T}$ is a quasicoherent injective $\mathscr{O}_{X}$-module. Let $\mathscr{K}_{\mathscr{P}}(\mathscr{T})^{\cdot}$ be the complex

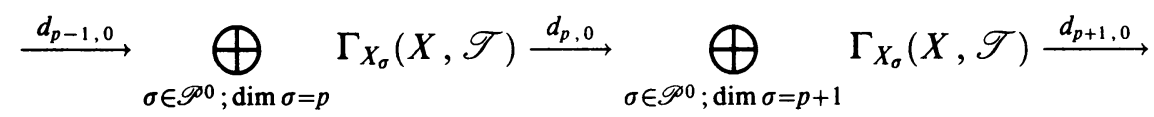

and let

$$
\varepsilon: \bigoplus_{\sigma \in \mathscr{P O} ; \operatorname{dim} \sigma=t} \Gamma_{X_{\sigma}}(X, \mathscr{T}) \rightarrow \Gamma_{X_{U}}(X, \mathscr{T})
$$

be

$$
\sum_{\operatorname{dim} \sigma=t} \beta_{\sigma} i_{\sigma, U}(\mathscr{F})
$$

Then

$$
0 \rightarrow \mathscr{K}_{\mathscr{P}}(\mathscr{T})^{\cdot} \rightarrow \Gamma_{X_{U}}(X, \mathscr{T}) \rightarrow 0
$$

is exact.

Proof. We will fix a particular $\mathscr{T}$ and denote $\mathscr{K}_{\mathscr{P}}(\mathscr{T})^{\bullet}$ by $\mathscr{K}_{\mathscr{P}}$.

It is clear that (6) is a complex. The proof that it is exact will be by induction on $m$. It will follow from the induction procedure described below that the starting cases are those where $U$ is itself an element of $\mathscr{P}$. In those cases $\mathscr{P}_{0}$ consists of a single element $U$ and hence the conclusion is obvious.

Define

$$
\begin{gathered}
U_{+}=\left\{\lambda \in U \mid\left\langle\lambda, \chi_{m}\right\rangle \geq 0\right\}, \quad U_{0}=\left\{\lambda \in U \mid\left\langle\lambda, \chi_{m}\right\rangle=0\right\}, \\
U_{-}=\left\{\lambda \in U \mid\left\langle\lambda, \chi_{m}\right\rangle \leq 0\right\} .
\end{gathered}
$$

Clearly $U_{+} \cup U_{-}=U, U_{+} \cap U_{-}=U_{0}$.

If $U_{+}=U$ or $U_{-}=U$ then we can define $\mathscr{P}^{0}$ using only $\chi_{1}, \ldots, \chi_{m-1}$. Hence the result follows by induction.

In the other case $\operatorname{dim} U_{+}=\operatorname{dim} U_{-}=\operatorname{dim} U_{0}+1=t$. We define $\mathscr{P}_{\varepsilon}=$ $\left\{\sigma \in \mathscr{P} \mid \sigma \subset U_{\varepsilon}\right\}$ where $\varepsilon= \pm, 0 . \mathscr{P}_{+}^{0}, \mathscr{P}_{-}^{0}, \mathscr{P}_{0}^{0}$ are defined as $\mathscr{P}^{0}$, but using $U_{+}, U_{-}, U_{0}$ instead of $U$.

Given $U_{ \pm}, U_{0}$, we can define $\mathscr{P}_{ \pm}^{0}, \mathscr{P}_{0}^{0}$ without using $\chi_{m}$. Hence for $U_{ \pm}$ and $U_{0}$ the conclusion is true by induction on $m$.

The inclusions $\mathscr{P}_{+}^{0}, \mathscr{P}_{-}^{0} \subset \mathscr{P}^{0}$ induce a map $\mathscr{K}_{\dot{\mathscr{P}}_{+}} \oplus \mathscr{K}_{\dot{\mathscr{P}}_{-}} \rightarrow \mathscr{K}_{\mathscr{P}}$. It is then easy to see that there is a short exact sequence of complexes

$$
0 \rightarrow \mathscr{K}_{\dot{\mathscr{P}}_{+}} \oplus \mathscr{K}_{\dot{\mathscr{P}}_{-}} \rightarrow \mathscr{K}_{\mathscr{P}} \rightarrow \mathscr{K}_{\dot{\mathscr{P}}_{0}} \rightarrow 0
$$

which gives rise to a long exact homology sequence. We obtain at once that $H^{q}\left(\mathscr{K}_{\mathscr{P}}\right)=0$ if $q \leq t-2$.

Furthermore we may construct a commutative diagram:

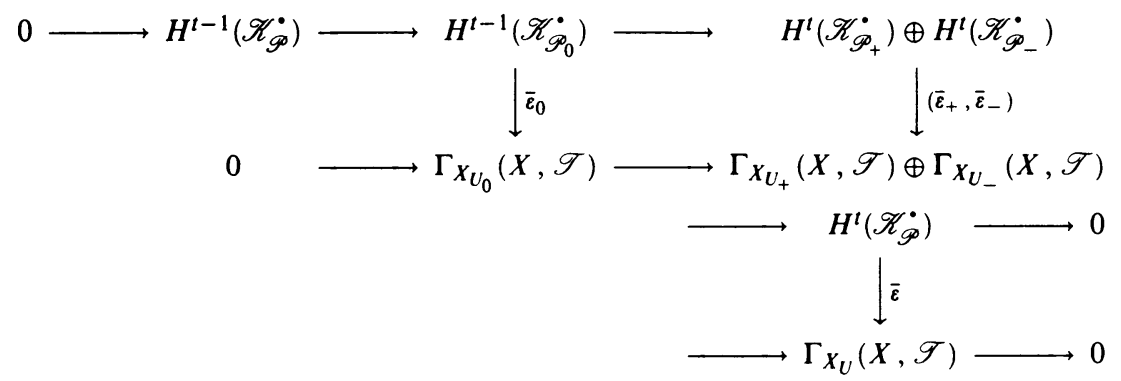


Here the lower sequence is obtained from the Mayer Vietoris sequence, using the fact that $X_{U}=X_{U_{+}} \cup X_{U_{-}}$and $X_{U_{0}}=X_{U_{+}} \cap X_{U_{-}}$by Lemma 3.1.2. This sequence is exact since $\mathscr{T}$ is injective.

$\varepsilon_{+}$and $\varepsilon_{-}$are defined as $\varepsilon$, but using $\left(\beta_{\sigma}\right)_{\sigma \in \mathscr{P}_{+}^{0} ; \operatorname{dim} \sigma=t},\left(\beta_{\sigma}\right)_{\sigma \in \mathscr{P}_{-}^{0} ; \operatorname{dim} \sigma=t}$ respectively.

The right most square in (7) is obviously commutative. Using this we may construct a map $\varepsilon_{0}$ which makes the left most square commutative. It is easy to compute that it has the form (5) (with different $\beta$ 's) and hence $\bar{\varepsilon}_{0}$ is an isomorphism using the induction hypothesis. Since by the induction hypothesis, $\bar{\varepsilon}_{+}, \bar{\varepsilon}_{-}$are also isomorphisms we deduce that $H^{t-1}\left(\mathscr{K}_{\mathscr{P}}\right)=0$ and that $\bar{\varepsilon}$ is an isomorphism.

Proof of Theorem 3.2.1. This is now standard. One starts with an injective resolution $0 \rightarrow \mathscr{F} \rightarrow \mathscr{T}^{\cdot}$ and makes the double complex $\mathscr{K}_{\mathscr{P}}\left(\mathscr{T}^{*}\right)^{\circ}$. After taking homology for the first filtration on this double complex, we obtain the required spectral sequence.

3.3 Cohomology with support in a linear subspace. It will become clear in the next subsection that, to apply (4), we have to be able to compute $H_{X_{\lambda}}\left(X, \mathscr{O}_{X}\right)$. This is what we will do here.

To compute $H_{X_{\lambda}}\left(X, \mathscr{O}_{X}\right)$ we use the fact that the defining ideal of $X_{\lambda}$ is generated by a subspace of $W$. Hence let $W^{\prime}$ be a subspace of $W$, spanned by some of the basis vectors $\left(w_{i}\right)_{i}$ and assume that $d^{\prime}=\operatorname{dim} W^{\prime}$. Put $W^{\prime \prime}=$ $W / W^{\prime}$ and let $J$ be the ideal generated by $W^{\prime}$. We want to describe $H_{J}^{i}(R)$ as a $\mathbb{Z}^{d}$ graded vector space (and hence as a $T$-module).

\section{Proposition 3.3.1.}

- $H_{J}^{i}(R)=0$ if $i \neq d^{\prime}$.

- $H_{J}^{d^{\prime}}(R)$ is, as a $\mathbb{Z}^{d}$ graded vector space, isomorphic to $\left(\bigwedge^{d^{\prime}} W^{\prime}\right)^{*} \otimes$ $\bigoplus_{n=0}^{\infty} S^{n}\left(W^{\prime *} \oplus W^{\prime \prime}\right)$.

Proof. The first statement is clear since $J$ is generated by a regular sequence.

For the second statement, we use the fact that

$$
H_{J}^{d^{\prime}}(R)=\underset{t}{\lim } \operatorname{Ext}_{R}^{d^{\prime}}\left(R / J^{t}, R\right) \text {. }
$$

We first compute

$$
\operatorname{Ext}^{i}\left(J^{t} / J^{t+1}, R\right) \cong\left(S^{t} W^{\prime}\right)^{*} \otimes \operatorname{Ext}_{R}^{i}(R / J, R) .
$$

Again $\operatorname{Ext}^{i}(R / J, R)=0$ if $i \neq d^{\prime}$. On the other hand, using the Koszul resolution, one easily computes that $\operatorname{Ext}_{R}^{d^{\prime}}(R / J, R) \cong\left(\bigwedge^{d^{\prime}} W^{\prime}\right)^{*} \otimes R / J$. Hence as $\mathbb{Z}^{d}$-graded vector space

$$
\begin{aligned}
H_{J}^{i}(R) & =\bigoplus_{t} \bigoplus_{t^{\prime}}\left(\bigwedge^{d^{\prime}} W^{\prime}\right)^{*} \otimes\left(S^{t} W^{\prime}\right)^{*} \otimes S^{t} W^{\prime \prime} \\
& =\left(\bigwedge^{d^{\prime}} W^{\prime}\right)^{*} \otimes \bigoplus_{t \geq 0} S^{t}\left(W^{\prime *} \oplus W^{\prime \prime}\right) .
\end{aligned}
$$

In the sequel if $a=\left(a_{1}, \ldots, a_{d}\right) \in \mathbb{Z}^{d}$ then supp $a$ will be $\left\{i \mid a_{i}<0\right\}$. Let $\lambda \in E^{*}$ and define $d_{\lambda}=d-\left|T_{\lambda}\right|$. 
Corollary 3.3.2.

- If $f \in H_{X_{\lambda}}^{d_{\lambda}}\left(X, \mathscr{O}_{X}\right)$ then supp_ $\operatorname{deg} f=T_{\lambda}^{c}$ where $c$ denotes the complement with respect to $\mathscr{W}$.

- $H_{X_{\lambda}}^{i}\left(X, \mathscr{O}_{X}\right)=0$ if $i \neq d_{\lambda}$ and the weights of $H_{X_{\lambda}}^{d_{\lambda}}\left(X, \mathscr{O}_{X}\right)$ are of the form

$$
-\sum_{i \in T_{\lambda}^{c}} \alpha_{i}-\sum_{i \in T_{\lambda}^{c}} a_{i} \alpha_{i}+\sum_{i \in T_{\lambda}} b_{i} \alpha_{i}
$$

where $a_{i}, b_{i} \in \mathbb{N}$.

3.4 Interpretation of the spectral sequence. In this section we retain the notations of the previous sections. We will show how the spectral sequence (4) leads to a description of $H_{X_{U}}\left(X, \mathscr{O}_{X}\right)$ in terms of one-parameter subgroups in $U$.

If $\lambda, \mu \in E^{*}$ then we say that $\lambda$ and $\mu$ are equivalent (notation $\lambda \sim \mu$ ) if $X_{\lambda}=X_{\mu}$ (or equivalently if $T_{\lambda}=T_{\mu}$ ). If $V \subset E^{*}$ then $V_{\lambda}=\{\mu \in V \mid \mu \sim$ $\lambda\}$. We assume from now on that $U=\bar{B}$. This restriction is convenient but immaterial for the arguments presented below. $B / \sim$ will be denoted by $\Lambda$.

We may define $\mathscr{P}$ as in $\S 3.2$. We will assume that the $\chi_{1}, \ldots, \chi_{m}$ are chosen in such a way that the elements of $\mathscr{P}$ define a structure of a pseudomanifold with boundary on $\bar{B}$. This means we we may choose $\alpha_{\sigma^{\prime}, \sigma}$ and $\beta_{\sigma}$, as explained in $\S 3.2$ after the statement of Theorem 3.2.1.

We will also assume that

$$
\left\{\alpha_{1}, \ldots, \alpha_{d}\right\} \subset\left\{\chi_{1}, \ldots, \chi_{m}\right\} .
$$

Then for every $\sigma \in \mathscr{P}^{0}$, relint $\sigma \subset B_{\lambda}$ for some $\lambda \in \sigma$. Hence $H_{X_{\sigma}}\left(X, \mathscr{O}_{X}\right)=$ $H_{X_{\text {relint } \sigma}}\left(X, \mathscr{O}_{X}\right)=H_{X_{\lambda}}^{\cdot}\left(X, \mathscr{O}_{X}\right)$ using Lemma 3.1.3.

Since $i_{\sigma \sigma^{\prime}}\left(\mathscr{O}_{X}\right)$ is a graded map, it follows from Corollary 3.3 .2 that if relint $\sigma \subset B_{\lambda}$, relint $\sigma^{\prime} \subset B_{\mu}$ where $\lambda \nsim \mu$ then $i_{\sigma^{\prime} \sigma}\left(\mathscr{O}_{X}\right)=0$. Hence $E_{p q}^{1}=\bigoplus_{\lambda \in \Lambda} E_{\lambda, p q}^{1}$ where

$$
E_{\lambda, p q}^{1}=\bigoplus_{\operatorname{relint} \sigma \subset B_{\lambda} ; \operatorname{dim} \sigma=p} H_{X_{\lambda}}^{q}\left(X, \mathscr{O}_{X}\right)
$$

and each $E_{\lambda}^{1}$ is closed under $d$. Hence we may write $d_{p q}=\bigoplus_{\lambda} d_{\lambda, p q}$ where $d_{\lambda, p q}$ goes from $E_{\lambda, p q}^{1}$ to $E_{\lambda, p+1 q}^{1}$.

Let $\Phi_{\lambda}=\overline{B_{\lambda}}-B_{\lambda}$. This is a CW-subcomplex of $\bar{B}$. From the description (9) it is easy to see that the complex $\left(E_{\lambda, p q}^{1}, d_{\lambda, p q}\right)$ has homology $H^{p}\left(\overline{B_{\lambda}}, \Phi_{\lambda}, k\right) \otimes$ $H_{X_{\lambda}}^{q}\left(X, \mathscr{O}_{X}\right)$. It also follows from Corollary 3.3.2 that if $i \neq j$ or $\lambda \neq \mu$ then any graded map between $H_{X_{\lambda}}^{i}\left(X, \mathscr{O}_{X}\right)$ and $H_{X_{\mu}}^{j}\left(X, \mathscr{O}_{X}\right)$ must be 0.

Hence one deduces that (4) degenerates at the $E^{2}$ term. Using this one obtains that there is a $T$-equivariant filtration on $H_{X^{u}}^{i}\left(X, \mathscr{O}_{X}\right)$ (as an $R$-module) such that

$$
\operatorname{gr} H_{X_{u}}^{i}\left(X, \mathscr{O}_{X}\right)=\bigoplus_{p+q=i+s ; \lambda \in \Lambda} H^{p}\left(\overline{B_{\lambda}}, \Phi_{\lambda}, k\right) \otimes_{k} H_{X_{\lambda}}^{q}\left(X, \mathscr{O}_{X}\right)
$$

which may be simplified to give the following theorem. 
Theorem 3.4.1. With notations as above

$$
\operatorname{gr} H_{X^{u}}^{i}\left(X, \mathscr{O}_{X}\right)=\bigoplus_{\lambda \in \Lambda} \tilde{H}^{i+s-d_{\lambda}-1}\left(\Phi_{\lambda}, k\right) \otimes H_{X_{\lambda}}^{d_{\lambda}}\left(X, \mathscr{O}_{X}\right) .
$$

Here we have used the standard convention $\widetilde{H}^{i}(\varnothing, k)=k$ if $i=-1$ and $\widetilde{H}^{i}(\varnothing, k)=0$ if $i \neq-1$.

Corollary 3.4.2. Assume that $a=\left(a_{1}, \ldots, a_{d}\right) \in \mathbb{Z}^{d}$. Then for $H_{\left(R^{T}\right)^{+}}^{i}\left(R_{\chi}^{T}\right)_{a}$ to be nonzero the following conditions must hold:

- $\chi=a_{1} \alpha_{1}+\cdots+a_{d} \alpha_{d}$

- $\exists \lambda \in Y(T)$ : supp_a $a=T_{\lambda}^{c}$ (such $a \lambda$ is unique up to equivalence);

- $0 \leq d_{\lambda}-i \leq s$.

In that case $H_{\left(R^{T}\right)^{+}}^{i}\left(R_{\chi}^{T}\right)_{a}$ will be isomorphic to $\tilde{H}^{i+s-d_{\lambda}-1}\left(\Phi_{\lambda}, k\right)$.

Recall that in [7] Stanley introduces for $a \in \mathbb{Z}^{d}$ polyhedral complexes $\Gamma_{a}$ (which only depend on supp $a$ ). The dimension of these complexes is in general of the order of $d$ while the dimension of the $\Phi_{\lambda}$ 's is of the order of $s$.

The main result is that if $\chi=a_{1} \alpha_{1}+\cdots+a_{d} \alpha_{d}$, then

$$
H_{\left(R^{T}\right)^{+}}^{i}\left(R_{\chi}^{T}\right)_{a} \cong \widetilde{H}_{h-i-1}\left(\Gamma_{a}, k\right) \text {. }
$$

We may then use Corollary 3.4 .2 to compute $\tilde{H}_{h-i-1}\left(\Gamma_{a}, k\right)$ in some cases.

Corollary 3.4.3. With notations as above. If there exists a $\lambda$ such that supp $a=$ $T_{\lambda}^{c}$, then $\widetilde{H}_{h-i-1}\left(\Gamma_{a}, k\right) \cong \widetilde{H}^{i+s-d_{\lambda}-1}\left(\Phi_{\lambda}, k\right)$. Otherwise $\Gamma_{a}$ is acyclic.

The following proposition gives some trivial observations about the $\Phi_{\lambda}$ 's. The point is that, if $\lambda \nsim 0$, we may replace $\Phi_{\lambda}$ with a subcomplex of the boundary complex of a spherical polytope.

\section{Proposition 3.4.4.}

(1) $\Phi_{0}$ is the empty set or a spherical polyhedral set (possibly the whole sphere).

(2) If $\lambda \nsim 0$ define $\Phi_{\lambda}^{\prime}=\overline{S_{\lambda}}-S_{\lambda}$. Then $\tilde{H}^{i}\left(\Phi_{\lambda}, k\right)=\tilde{H}^{i-1}\left(\Phi_{\lambda}^{\prime}, k\right)$.

Proof. (1) is easy. To prove (2) define the following sets

$$
\Phi^{(1)}=\left\{\mu \in \Phi_{\lambda} \mid\|\mu\| \geq 1 / 4\right\}, \quad \Phi^{(2)}=\left\{\mu \in \Phi_{\lambda} \mid\|\mu\| \leq 3 / 4\right\} .
$$

Then $\Phi^{(2)}$ is contractible. $\Phi^{(1)}$ is homotopy equivalent with a spherical polytope. Hence $\Phi^{(1)}$ is also contractible. Furthermore $\Phi^{(1)} \cap \Phi^{(2)}$ is homotopy equivalent with $\Phi_{\lambda}^{\prime}$. Since $\Phi_{\lambda}=\Phi^{(1)} \cup \Phi^{(2)}$, the result now follows from Mayer Vietoris.

Let $C_{0}$ be the apex set of $\operatorname{pos}\left\{\left(\alpha_{i}\right)_{i=1, \ldots, d}\right\}$ and let $c=\operatorname{dim} C_{0}$. Then the set

$$
\left\{\lambda \in B \mid\left\langle\lambda, \alpha_{i}\right\rangle=0 \text { if } \alpha_{i} \in C_{0} \text { and }\left\langle\lambda, \alpha_{i}\right\rangle<0 \text { otherwise }\right\}
$$

represents a single equivalence class which we will denote by $\bar{\lambda}_{n}$. The following, somewhat technical result, will be used later. 


\section{Proposition 3.4.5.}

(1) $\Phi_{\lambda_{n}}$ is homeomorphic to a $s-c-1$-dimensional sphere.

(2) If $i \neq h$ then $\widetilde{H}^{i+s-d_{\lambda_{n}}-1}\left(\Phi_{\lambda_{n}}, k\right)=0$; i.e., the term involving $\Phi_{\lambda_{n}}$ on the right-hand side of $(10)$ does not contribute to $H_{X^{u}}^{i}\left(X, \mathscr{O}_{X}\right)$ for $i \neq h$.

Proof. (1) is clear by inspection. (2) depends on the following formula, which is also easy to verify:

$$
h=\left|\left\{i \mid \alpha_{i} \in C_{0}\right\}\right|-c=d_{\lambda_{n}}-c .
$$

Hence if $\tilde{H}^{i+s-d_{\lambda_{n}}-1}\left(\Phi_{\lambda_{n}}, k\right) \neq 0$ then $i+s-d_{\lambda_{n}}-1=s-c-1$ which is equivalent with $i=d_{\lambda_{n}}-c=h$.

3.5 A new interpretation of $\Phi_{\lambda}^{\prime}$. Our aim is to give a new interpretation of the $\Phi_{\lambda}^{\prime}$ (as defined in Proposition 3.4.4). This will make it easier to visualize what the structure of $\Phi_{\lambda}^{\prime}$ is.

For $\lambda \in E^{*}$ define

$$
\begin{gathered}
A_{\lambda}=\operatorname{pos}\left\{\left(\alpha_{i}\right)_{i \in T_{\lambda}}\right\}, \quad E_{\lambda}=\operatorname{span}\left\{\left(\alpha_{i}\right)_{i \in T_{\lambda}}\right\}, \\
B_{\lambda}=\operatorname{pos}\left\{\left(\alpha_{i}\right)_{i \in T_{\lambda}},\left(-\alpha_{i}\right)_{i \notin T_{\lambda}}\right\} .
\end{gathered}
$$

We start with a few lemmas that will be needed later.

Lemma 3.5.1. Assume that $T$ acts faithfully on $W$. Then $\operatorname{relint} B_{\lambda}=\operatorname{int} B_{\lambda}$.

Proof. This follows from the definition of relint and the fact that $B_{\lambda}$ spans $E$ (using (2)).

Lemma 3.5.2. Assume that $T$ acts faithfully on $W$. Then

$$
\left\{\left(\alpha_{i}\right)_{i \in T_{\lambda}}\right\} \subset \partial \operatorname{pos}\left\{\left(\alpha_{i}\right)_{i=1, \ldots, d}\right\} \Leftrightarrow E_{\lambda} \cap \text { int } B_{\lambda}=\varnothing .
$$

Proof. $(\Leftarrow)$ Suppose that $E_{\lambda} \cap$ int $B_{\lambda}=\varnothing$. This means by Lemma 3.5.1 that $E_{\lambda}$ only hits $\partial B_{\lambda}$. We may then extend $E_{\lambda}$ to a supporting hyperplane $H$ for $B$. Then $\left(\alpha_{i}\right)_{i \in T_{\lambda}},\left(-\alpha_{i}\right)_{i \notin T_{\lambda}}$ all lie on one side of $H$, but since the $\left(\alpha_{i}\right)_{i \in T_{\lambda}}$ lie in $H$ we conclude that $\left(\alpha_{i}\right)_{i=1, \ldots, d}$ all lie on the same side of $H$. Hence $\left\{\left(\alpha_{i}\right)_{i \in T_{\lambda}}\right\} \subset \partial \operatorname{pos}\left\{\left(\alpha_{i}\right)_{i=1, \ldots, d}\right\}$.

$(\Rightarrow)$ To prove this direction we reverse the above argument.

Lemma 3.5.3. $A_{\lambda} \cap$ apex $B_{\lambda}=0$.

Proof. The apex of $B_{\lambda}$ must lie in the hyperplane defined by $\lambda$. This hyperplane intersects $A_{\lambda}$ only in 0 . This proves what we want.

The following theorem will be our main result.

Theorem 3.5.4. Assume that $T$ acts faithfully on $W$. Then $\Phi_{\lambda}^{\prime}$ has the same homotopy type as $A_{\lambda} \cap \partial B_{\lambda} \cap S$.

Proof. Let $A_{1}=A_{\lambda} \cap S, B_{1}=B_{\lambda} \cap S$. Recall that if $U_{1}$ is a spherical polyhedral set then $U_{1}^{*}$ is defined as $\{\mu \in S \mid \forall u \in U:\langle\mu, u\rangle \leq 0\}$.

The lattice of faces of $U_{1}$ is the opposite of the lattice of faces of $U_{1}^{*}$. For a face $\mathscr{F}$ of $U_{1}$ the corresponding face $\mathscr{F} \circ$ of $U_{1}^{*}$ is given by

$$
\mathscr{F}^{\circ}=\left\{\lambda \in U_{1}^{*} \mid \forall u \in \mathscr{F}:\langle\lambda, u\rangle=0\right\} \text {. }
$$


Similarly $\mathscr{F}=\left\{u \in U_{1} \mid \forall \lambda \in \mathscr{F}^{\circ}:\langle\lambda, u\rangle=0\right\}$. From this definition it follows that

$$
\left(\operatorname{apex} U_{1}\right)^{\circ}=U_{1}^{*} \text {. }
$$

We may now define $S_{\lambda}$ as $\left\{\mu \in B_{1}^{*} \mid \forall a \in A_{1}:\langle\mu, a\rangle<0\right\}$.

Claim 1. relint $B_{1}^{*} \subset S_{\lambda} \subset B_{1}^{*}$. We have to prove that $B_{1}^{*} \backslash S_{\lambda} \subset \partial B_{1}^{*}$. Let $\mu \in B_{1}^{*} \backslash S_{\lambda}$. Then there is some $a \in A_{1}$ such that $\langle\mu, a\rangle=0$.

By Lemma 3.5.3 $a$ is not an apex of $B_{1}$. Hence the hyperplane defined by $\mu$ intersects $B_{1}$ in a point which is not an apex. Therefore $\mu \in \partial B_{1}^{*}$ (using (12)).

Consequently $\Phi_{\lambda}^{\prime}=\overline{S_{\lambda}}-S_{\lambda}=B_{1}^{*}-S_{\lambda} \subset \partial B_{1}^{*}$, and hence

$$
\Phi_{\lambda}^{\prime}=\left\{\mu \in \partial B_{1}^{*} \mid \exists a \in A_{1}:\langle\mu, a\rangle=0\right\} .
$$

Define the following subcomplex of $\partial B_{1}^{*}$ :

$$
\Delta=\left\{\mathscr{F}^{\circ} \mid \mathscr{F} \text { face of } \partial B_{1}, \mathscr{F} \cap A_{1} \neq \varnothing\right\} .
$$

Claim 2. $|\Delta|=\bigcup_{\mathscr{F} \circ \in \Delta} \mathscr{F} \circ=\Phi_{\lambda}^{\prime}$. Let $\mu \in \mathscr{F}^{\circ}$ and choose $a \in \mathscr{F} \cap A_{1}$. Then $a$ is an element of $A_{1}$ such that $\langle\mu, a\rangle=0$ (11). Therefore by (13) $\mu \in \Phi_{\lambda}^{\prime}$.

Conversely let $\mu \in \Phi_{\lambda}^{\prime}$. Then by (13) there exists an $a \in A_{1}$ such that $\langle\mu, a\rangle=0$. By 3.5.1 $\mu$ cannot be everywhere 0 on $B_{1}$ and hence $a \in \partial B_{1}$. Let $\mathscr{F}$ be the smallest face of $\partial B_{1}$ containing $a$. then $\mathscr{F}^{\circ} \in \Delta$ and $\mu$ must also vanish on $\mathscr{F}$. Therefore $\mu \in \mathscr{F}^{\circ}$ (11). Now let $\mu^{\prime} \in \mathscr{F}^{\circ}$ be arbitrary. Then $\mu^{\prime}$ vanishes on $\mathscr{F}$ and in particular on $a$. Therefore $\mu^{\prime} \in \Phi_{\lambda}^{\prime}$ and we have shown that $\mu \in \mathscr{F}^{\circ} \subset \Phi_{\lambda}$. This proves our claim.

Let $L(\Delta)$ resp. $L\left(A_{1} \cap \partial B_{1}\right)$ be the lattices of faces of $\Delta$ and $A_{1} \cap \partial B_{1}$. Then there exists a map $\phi: L(\Delta) \rightarrow L(A \cap \partial B)^{\text {opp }}: \mathscr{F} \circ \mapsto \mathscr{F} \cap A_{1}$.

Furthermore if $\mathscr{G} \in L(A \cap \partial B)^{\text {opp }}$ then (as in [4, p. 93])

$$
\phi / \mathscr{G}=\left\{F^{\circ} \mid \phi(\mathscr{F} \circ) \leq \mathscr{G}\right\}=\left\{\mathscr{F} \circ \mathscr{F} \cap A_{1} \supset \mathscr{G}\right\} .
$$

Define $\mathscr{G}^{\prime}=\bigcap_{\mathscr{F} \cap A_{1} \supset \mathscr{F}^{\mathscr{F}}}$. Then $\mathscr{G}^{\prime \circ} \in L(\Delta)$ and $\phi / \mathscr{G}=\left\{\mathscr{F} \mid \mathscr{F} \supset \mathscr{G}^{\prime}\right\}=$ $\left\{\mathscr{F} \circ \mid \mathscr{G}^{\prime \circ} \supset \mathscr{F} \circ\right\}$ is contractible and hence by [4, Theorem A, p. 93] $\phi$ induces a homotopy equivalence between $|L(\Delta)|$ and $\left|L\left(A_{1} \cap \partial B_{1}\right)^{\text {opp }}\right|$.

Since $\Phi_{\lambda}^{\prime}=|L(\Delta)|$ and

$$
\left|L\left(A_{1} \cap \partial B_{1}\right)^{\text {opp }}\right| \cong\left|L\left(A_{1} \cap \partial B_{1}\right)\right| \cong A_{1} \cap \partial B_{1},
$$

we are done.

3.6 The structure of some special $\Phi_{\lambda}^{\prime}$. In general, the structure of $\Phi_{\lambda}^{\prime}$ can be arbitrarily complicated in the sense that apart from the fact that $\Phi_{\lambda}^{\prime}$ should be a subcomplex of the boundary complex of a spherical polytope, there are no other restrictions. However for some special $\lambda$ 's the structure of $\Phi_{\lambda}^{\prime}$ can be described.

On $\Lambda-\{\overline{0}\}$ we define a partial ordering as follows:

$$
\bar{\lambda}_{1} \leq \bar{\lambda}_{2} \Leftrightarrow \text { relint } \operatorname{pos}\left\{\left(\alpha_{i}\right)_{i \in T_{\lambda_{1}}}\right\} \subset \text { relint } \operatorname{pos}\left\{\left(\alpha_{i}\right)_{i \in T_{\lambda_{2}}}\right\} \text {. }
$$

Then it turns out that for the maximal elements under this ordering $\Phi_{\lambda}^{\prime}$ is either contractible or homotopy equivalent with a sphere.

The following proposition shows that $\leq$ does indeed define a partial ordering on $\Lambda-\{\overline{0}\}$. 
Proposition 3.6.1. If $\bar{\lambda}_{1,2} \in \Lambda-\{\overline{0}\}, \bar{\lambda}_{1} \leq \bar{\lambda}_{2}$ then $T_{\lambda_{1}} \subset T_{\lambda_{2}}$.

Proof. By taking closures, it follows from (14) that $\bar{\lambda}_{1} \leq \bar{\lambda}_{2}$ implies that $\forall i \in$ $T_{\lambda_{1}}: \alpha_{i} \in \operatorname{pos}\left\{\left(\alpha_{j}\right)_{j \in T_{\lambda_{2}}}\right\}$.

Fix $i \in T_{\lambda_{1}}$. Since $\alpha_{i} \neq 0, \alpha_{i}$ must be strictly positive linear combinations of some of the $\left(\alpha_{j}\right)_{j \in T_{\lambda_{2}}}$. Hence $\left\langle\lambda_{2}, \alpha_{i}\right\rangle<0$ and therefore $i \in T_{\lambda_{2}}$.

Corollary 3.6.2. If $\bar{\lambda}_{1} \leq \bar{\lambda}_{2}$ and $\bar{\lambda}_{2} \leq \bar{\lambda}_{1}$ then $\lambda_{1} \sim \lambda_{2}$.

We will prove the following fact.

Theorem 3.6.3. Assume that $T$ acts faithfully on $W$. Let $\lambda$ be maximal with respect to the partial ordering defined on $\Lambda-\{\overline{0}\}$. Then

1. If $\left\{\left(\alpha_{i}\right)_{i \in T_{\lambda}}\right\} \subset \partial \operatorname{pos}\left\{\left(\alpha_{i}\right)_{i=1, \ldots, d}\right\}$ then $\Phi_{\lambda}^{\prime}$ is contractible and hence $\widetilde{H}^{i}\left(\Phi_{\lambda}^{\prime}, k\right)=0$ for all $i$;

2. Otherwise, $\Phi_{\lambda}^{\prime}$ is homotopic to a $u_{\lambda}-2$ dimensional sphere where $u_{\lambda}=$ $\operatorname{dim} E_{\lambda}$. Hence

$$
\widetilde{H}^{i}\left(\Phi_{\lambda}^{\prime}, k\right)= \begin{cases}k & \text { if } i=u_{\lambda}-2, \\ 0 & \text { otherwise. }\end{cases}
$$

Recall that $E_{\lambda}$ was defined in the beginning of $\S 3.5$.

The proof of Theorem 3.6.3 will be based upon a series of lemmas.

Lemma 3.6.4. Assume that we have chosen $T \subset\{1, \ldots, d\}, \mu \in E^{*}-\{0\}$ such that $T \subset T_{\mu}$. Assume furthermore that there exists

$$
y \in E_{\mu} \cap \operatorname{pos}\left\{\left(\alpha_{i}\right)_{i \notin T}\right\}
$$

with the property that $\langle\mu, y\rangle<0$. Then there exist $\lambda \in E^{*}-\{0\}, T^{\prime} \subset$ $\{1, \ldots, d\}$ with the property that

1. $T \subsetneq T^{\prime} \subset T_{\lambda}$ and

2. relint $\operatorname{pos}\left\{\left(\alpha_{i}\right)_{i \in T}\right\} \subset$ relint $\operatorname{pos}\left\{\left(\alpha_{i}\right)_{i \in T^{\prime}}\right\}$.

Proof. Choose $y \in E_{\mu} \cap \operatorname{pos}\left\{\left(\alpha_{i}\right)_{i \notin T}\right\}$ such that $y$ may be written as

$$
y=\sum_{i \in T^{\prime \prime}} a_{i} \alpha_{i}, \quad \text { all } a_{i}>0
$$

with the smallest possible $T^{\prime \prime} \subset T^{c}$ such that $\langle\mu, y\rangle<0$. In particular $T^{\prime \prime} \neq$ $\varnothing$.

Choose $z \in$ relint $\operatorname{pos}\left\{\left(\alpha_{i}\right)_{i \in T}\right\}$, i.e.,

$$
z=\sum_{i \in T} b_{i} \alpha_{i}, \quad \text { all } b_{i}>0 .
$$

Since also $y \in E_{\lambda}$ we may also choose $\left(a_{i}\right)_{i \in T} \in \mathbb{R}$ such that

$$
y=\sum_{i \in T}\left(-a_{i}\right) \alpha_{i} .
$$

Define $T^{\prime}=T \cup T^{\prime \prime}$. Subtracting (16) from (15) yields $0=\sum_{i \in T^{\prime}} a_{i} \alpha_{i}$. Then, for $t>0$ small enough, the expression

$$
z=\sum_{i \in T}\left(b_{i}+t a_{i}\right) \alpha_{i}+\sum_{i \in T^{\prime \prime}} t a_{i} \alpha_{i}
$$

will have positive coefficients. This shows that $z \in$ relint $\operatorname{pos}\left\{\left(\alpha_{i}\right)_{i \in T^{\prime}}\right\}$ which proves 2 and part of 1 . 
To verify the existence of $\lambda$ we have to show that

$0 \notin \operatorname{relint} \operatorname{pos}\left\{\left(\alpha_{i}\right)_{i \in T^{\prime}}\right\}$.

Assume that there are $\left(u_{i}\right)_{i \in T^{\prime}}$ such that

$$
0=\sum_{i \in T^{\prime}} u_{i} \alpha_{i}, \quad \text { all } u_{i}>0
$$

Then for $t>0$,

$$
y^{\prime}=y-t \sum_{i \in T^{\prime \prime}} u_{i} \alpha_{i}
$$

still lies in $E_{\mu}$ and has the property that $\left\langle\mu, y^{\prime}\right\rangle<0$.

Moreover, if we choose $t=\min _{i \in T^{\prime \prime}} a_{i} / u_{i}$ then $y^{\prime}=\sum_{i \in T^{\prime \prime}}\left(a_{i}-t u_{i}\right) \alpha_{i}$ is in $\operatorname{pos}\left\{\left(\alpha_{i}\right)_{i \notin T}\right\}$ and has smaller support than $y$. This contradicts the choice of $y$.

Lemma 3.6.5. Let $T \subset\{1, \ldots, d\}, \mu \in E^{*}-\{0\}$ be such that $T \subset T_{\mu}$. Then there exist $\lambda \in E^{*}-\{0\}$ such that

$$
\text { relint } \operatorname{pos}\left\{\left(\alpha_{i}\right)_{i \in T}\right\} \subset \text { relint } \operatorname{pos}\left\{\left(\alpha_{i}\right)_{i \in T_{\lambda}}\right\} .
$$

Proof. Assume that the result is false and let $(T, \mu)$ be a counterexample with $T$ maximal.

Let $E_{\mu 0}=E_{\mu} \cap \operatorname{ker} \mu$. By the previous lemma we may assume that for all $y \in E_{\mu} \cap \operatorname{pos}\left\{\left(\alpha_{i}\right)_{i \notin T}\right\}$ it is true that $\langle\mu, y\rangle \geq 0$; i.e., $E_{\mu} \cap \operatorname{pos}\left\{\left(\alpha_{i}\right)_{i \notin T}\right\}$ lies on one side of $E_{\mu 0}$ whereas the $\left(\alpha_{i}\right)_{i \in T}$ lie strictly on the other side.

It is then easy to see that we may extend $E_{\mu 0}$ to a hyperplane in $E$, separating $\left(\alpha_{i}\right)_{i \in T}$ from $\left(\alpha_{i}\right)_{i \notin T}$. Hence $T=T_{\lambda}$. This contradicts the fact that $(T, \mu)$ was a counterexample.

Lemma 3.6.6. Let $\lambda \in \Lambda-\{0\}$ be maximal for the partial ordering defined by (14). Then $A_{\lambda}=B_{\lambda} \cap E_{\lambda}$.

Proof. Certainly $A_{\lambda} \subset B_{\lambda} \cap E_{\lambda}$. To prove the converse let

$$
\beta_{i}= \begin{cases}\alpha_{i} & \text { if } i \in T_{\lambda}, \\ -\alpha_{i} & \text { if } i \notin T_{\lambda} .\end{cases}
$$

Let $y$ be the element of $B_{\lambda} \cap E_{\lambda} \backslash A_{\lambda}$ which can be expressed in the form

$$
y=\sum_{i \in T} a_{i} \beta_{i}, \quad \text { all } a_{i}>0,
$$

with smallest possible $T$. Note that $\beta_{i} \neq 0$ for $i \in T \cup T_{\lambda}$.

Claim. $\operatorname{pos}\left\{\left(\beta_{i}\right)_{i \in T}\right\} \cap A_{\lambda}=\{0\}$. Suppose not. Then there exist $z=\sum_{i \in T} b_{i} \beta_{i}$ $\in A_{\lambda}$ with $b_{i} \geq 0$ and not all $b_{i}=0$.

Let $t=\min _{i \in T} a_{i} / b_{i}$. Then $y^{\prime}=y-t z$ has smaller support than $y$. Furthermore, since $y=y^{\prime}+t z$, we see that $y^{\prime} \in B_{\lambda} \cap E_{\lambda} \backslash A_{\lambda}$, contradicting the minimality of $T$. This proves our claim. In particular $T \cap T_{\lambda}=\varnothing$.

Using the Claim, we may now choose a $\mu \in E^{*}-\{0\}$ strictly separating $\left(\beta_{i}\right)_{i \in T}$ and $\left(\beta_{i}\right)_{i \in T_{\lambda}}$; i.e.,

$$
\forall i \in T_{\lambda}:\left\langle\mu, \beta_{i}\right\rangle<0, \quad \forall i \in T:\left\langle\mu, \beta_{i}\right\rangle>0 .
$$

Hence $T_{\lambda} \subset T_{\mu}$. 
We deduce that

$$
-y=\sum_{i \in T} a_{i} \alpha_{i} \in E_{\lambda} \cap \operatorname{pos}\left\{\left(\alpha_{i}\right)_{i \in T_{\lambda}^{c}}\right\}
$$

has the property that $\langle\mu,-y\rangle<0$.

But then by Lemma 3.6.4 and Lemma 3.6.5 there exists $\lambda^{\prime} \in E^{*}-\{0\}$ such that $T_{\lambda} \subsetneq T_{\lambda^{\prime}}$ and

$$
\text { relint } \operatorname{pos}\left\{\left(\alpha_{i}\right)_{i \in T_{\lambda}}\right\} \subset \text { relint } \operatorname{pos}\left\{\left(\alpha_{i}\right)_{i \in T_{\lambda^{\prime}}}\right\} .
$$

But since $\lambda$ was chosen to be maximal under the partial ordering defined by (14), we obtain a contradiction.

Proof of Theorem 3.6.3. This is now a simple application of Theorem 3.5.4, Lemma 3.5.1 and Lemma 3.5.2.

In the first case $\Phi_{\lambda}^{\prime}$ will have the homotopy type of $A_{\lambda} \cap S$ which is contractible.

In the second case $\Phi_{\lambda}^{\prime}$ will have the homotopy type of $\partial A_{\lambda} \cap S$ which is a $u_{\lambda}-2$ dimensional sphere.

\section{Applications}

In this section we retain the notations of the previous sections.

We will give some applications of Theorem 3.4.1. However, before we continue, it is useful to introduce the concept of stability.

A point $x \in X$ is said to be stable if for all $\lambda \in Y(T)-\{0\}$ neither $\lim _{t \rightarrow 0} \lambda(t) x$ nor $\lim _{t \rightarrow \infty} \lambda(t) x$ exists. Stable points have finite stabilizer and closed $T$-orbit. They form an invariant open subset of $X$. One deduces that if $X$ has a stable point then $d=\operatorname{dim} R=\operatorname{dim} R^{T}+\operatorname{dim} T=h+s$. It is easy to see that $X$ has a stable point if and only if for all $\lambda \in Y(T)-\{0\}$ there exists an $i$ such that $\left\langle\lambda, \alpha_{i}\right\rangle>0$. This means that the weights of $W$ do not lie in a halfspace defined by a hyperplane in $E$ going through the origin.

It will become clear below that 3.4.1 is best suited for cases where $X$ has a stable point. This is the most interesting situation from a geometric viewpoint. Of course other cases can also be treated but then things are not as natural.

$4.1 \operatorname{dim} T=1$ or 2 . As a first application we note that it is easy to eyeball what the possible $\Phi_{\lambda}$ 's are if $\operatorname{dim} T$ is small.

Let us first consider the case $\operatorname{dim} T=1$. To avoid triviality we assume that $T$ acts faithfully on $X$. There are now two possibilities.

- $X$ does not have a stable point. This is the trivial case. We obtain that $\Lambda=\{\overline{0}, \bar{\lambda}\}$ where $\Phi_{0}$ consists of one point and $\Phi_{\lambda}$ consists of two points. Hence $H_{X^{u}}^{i}\left(X, \mathscr{O}_{X}\right)=H_{X_{\lambda}}^{d_{\lambda}}\left(X, \mathscr{O}_{X}\right)^{\delta_{i d_{\lambda}}}$ where $\delta$ is the Kronecker delta. One obtains, using [2, Satz 4.10 and 4.12], that if $R_{\chi}^{T} \neq\{0\}$ then $\operatorname{dim} R_{\chi}^{T}=d_{\lambda}$ and $R_{\chi}^{T}$ is Cohen-Macaulay. Of course this can be verified directly without any difficulty.

- $X$ does have a stable point. In this case $\Lambda=\left\{\overline{0}, \overline{\lambda_{1}}, \overline{\lambda_{2}}\right\}$ where $\Phi_{0}=\varnothing$ and $\Phi_{\lambda_{1}} \cong \Phi_{\lambda_{2}} \cong 2$ points. If we put this information in Corollary 3.4.2, we obtain a simple proof for [7, Theorem 3.3 and Corollary 3.4 ].

The conclusion of Theorem 3.4.1 may be written as

(18) $\operatorname{gr} H_{X^{u}}^{i}\left(X, \mathscr{O}_{X}\right)=H_{X_{\lambda_{1}}}^{d_{\lambda_{1}}}\left(X, \mathscr{O}_{X}\right)^{\delta_{i d_{1}}} \oplus H_{X_{\lambda_{2}}}^{d_{\lambda_{2}}}\left(X, \mathscr{O}_{X}\right)^{\delta_{i d_{2}}} \oplus H_{\{0\}}^{d}\left(X, \mathscr{O}_{X}\right)^{\delta_{i d-1}}$. 
TABLE 1

\begin{tabular}{|c|c|c|c|c|}
\hline Type & $\Phi_{\lambda}$ & \multicolumn{3}{|c|}{$\operatorname{dim} \tilde{H}^{i}\left(\Phi_{\lambda}, k\right)$} \\
\hline 1 & $\varnothing$ & $i=-1$ & $i=0$ & $i=1$ \\
\hline & $\bullet$ & 0 & 0 & 0 \\
\hline 2 & & 1 & 0 & 0 \\
\hline 3 & $\bullet$ & 0 & 1 & 0 \\
\hline 4 & $\bullet$ & 0 & 0 & 0 \\
\hline 5 & $\bullet$ & 0 & 0 & 0 \\
\hline 7 & $<$ & 0 & 0 & 1 \\
\hline
\end{tabular}

Let us now consider the case $\operatorname{dim} T=2$. We will assume that $T$ acts faithfully on $W$. In that case we can construct Table 1 (we have used an iconic representation of $\Phi_{\lambda}$ which we hope is sufficiently clear). Note that types 2 and 3 only occur if $X$ has no stable point.

Corollary 3.4.2 together with Table 1 gives now a complete description of the groups $H_{\left(R^{T}\right)^{+}}\left(R_{\chi}^{T}\right)$ if $\operatorname{dim} T=2$. We will illustrate this in the case that $X$ has a stable point. It follows from Table 1 that if $\lambda \neq 0$ then $\Phi_{\lambda}^{\prime}$ is either the empty set, one point or two points. Hence we may define a partition by $\Lambda-\{0\}=\Lambda_{\varnothing} \cup \Lambda . \cup \Lambda \ldots$. Then there is the following analog to [8, Corollary 3.4].

Corollary 4.1.1. Assume that $X$ has a stable point. Let $\mathscr{U}^{x} \subset \mathbb{Z}^{d}$ be the set of all integer solutions to $\sum_{i=1}^{d} a_{i} \alpha_{i}=\chi$, and define

$$
\Lambda_{\varnothing}^{\chi}=\left\{\bar{\lambda} \in \Lambda_{\varnothing} \mid \exists a \in \mathscr{U}^{\chi}: \operatorname{supp}_{-} a=T_{\lambda}^{c}\right\}
$$


and similarly

$$
\Lambda_{. .}^{\chi}=\left\{\bar{\lambda} \in \Lambda . . \mid \exists a \in \mathscr{U}^{\chi}: \operatorname{supp}_{-} a=T_{\lambda}^{c}\right\} .
$$

Assume that $R_{\chi}^{T} \neq 0$. Then

$$
\operatorname{depth} R_{\chi}^{T}=\min \left\{\left(d_{\lambda}-1\right)_{\lambda \in \Lambda_{\varnothing}^{\chi}},\left(d_{\lambda}\right)_{\lambda \in \Lambda_{. .}^{x}}\right\} .
$$

In particular $R_{\chi}^{T}$ is Cohen-Macaulay if and only if

$$
\left|T_{\lambda}\right| \leq 1 \text { for all } \bar{\lambda} \in \Lambda_{\varnothing}^{\chi} \text { and }\left|T_{\lambda}\right| \leq 2 \text { for all } \bar{\lambda} \in \Lambda_{. .}^{\chi} \text {. }
$$

Proof. This is a direct consequence of Corollary 3.4.2. For the criterion for Cohen-Macaulayness we use the fact that $d_{\lambda}=d-\left|T_{\lambda}\right|$ and $h=d-2$ (using the stability hypothesis).

Remark 4.1.2. This criterion is particularly simple to apply if for all $\bar{\lambda} \in \Lambda_{\varnothing}$ : $\left|T_{\lambda}\right|>1$ and for all $\bar{\lambda} \in \Lambda$..: $\left|T_{\lambda}\right|>2$. In that case $R_{\chi}^{T}$ will be Cohen-Macaulay if and only if

$$
\forall \bar{\lambda} \in \Lambda_{\varnothing} \cup \Lambda . .: \nexists a \in \mathscr{U}^{\chi}: \operatorname{supp}_{-} a=T_{\lambda}^{c} .
$$

To finish this section, we will give a criterion to decide whether $\bar{\lambda} \in \Lambda-\{0\}$ belongs to $\Lambda_{\varnothing}$, to $\lambda$. or to $\Lambda .$. . Note that $\Lambda-\{0\}=S / \sim$.

Let us call $\lambda \in S$ a local maximum if there exists an arc $\left[\lambda_{1}, \lambda_{2}\right] \subset S$ containing $\lambda$ in its interior, such that for all $\mu \in\left[\lambda_{1}, \lambda_{2}\right], d_{\mu} \leq d_{\lambda}$, and $d_{\lambda_{1}}, d_{\lambda_{2}}<d_{\lambda}$. A local minimum is defined similarly. The following is easy to see.

Proposition 4.1.3. If $\lambda \in S$ is a local maximum then $\bar{\lambda}$ belongs to $\Lambda_{\varnothing}$. If $\lambda$ is a local minimum then $\bar{\lambda}$ belongs to $\Lambda$.. . In the remaining case, $\bar{\lambda}$ belongs to $\Lambda$.

4.2 Relations with one-parameter subgroups. If $\lambda \in Y(T)$ then $\operatorname{im} \lambda$ is a subtorus of $T$. We will denote this subtorus also by $\lambda$. From Theorem 3.4.1 it is clear that the Cohen-Macaulayness of $R_{\chi}^{T}$ is related to the one-parameter subgroups of $T$. In fact one can prove the following result.

Theorem 4.2.1. Assume that $R_{\chi \circ \lambda}^{\lambda}$ is Cohen-Macaulay for all $Y(T) \backslash\{0\}$. Then $R_{\chi}^{T}$ is Cohen-Macaulay.

Proof. First assume that $X$ has a stable point (this is the easiest case). From the fact that $R_{\chi \circ \lambda}^{\lambda}$ is Cohen-Macaulay for all $\lambda \in Y(T) \backslash\{0\}$ we deduce from (18) that if $d_{\lambda}<d-1$ then $H_{X_{\lambda}}^{d_{\lambda}}\left(X, \mathscr{O}_{X}\right)_{\chi}^{T}=0$. Hence the only terms that can contribute to the right-hand side of (10) are those where $\lambda=0$ and those where $\lambda \neq 0, d_{\lambda} \geq d-1$.

Assume for some $i: \widetilde{H}^{i+s-d_{\lambda}-1}\left(\Phi_{\lambda}, k\right) \otimes H_{X_{\lambda}}^{d_{\lambda}}\left(X, \mathscr{O}_{X}\right)_{\chi}^{T} \neq 0$. If $\lambda=0$ we have that $i+s-d_{0}-1=-1$ or $i=d-s$. If $\lambda \neq 0$ then $\Phi_{\lambda} \neq \varnothing$ and hence $i+s-d_{\lambda}-1 \geq 0$. Combining this with $d_{\lambda} \geq d-1$ yields $i \geq d-s$. Therefore $H_{X^{u}}^{i}\left(X, \mathscr{O}_{X}\right)_{\chi}^{T}=0$ if $i<d-s=h$ and hence $R_{\chi}^{T}$ must be Cohen-Macaulay.

Now let us treat the general case. Using induction on $\operatorname{dim} T$ we will reduce to the stable case.

For $R_{\chi}^{T}$ to be nonzero, it is necessary that $\chi$ factors through the image of $T$ in $\operatorname{End}_{k}(W)$. Hence we will assume this (otherwise the theorem is vacuous). 
We then replace $T$ with its image in $\operatorname{End}(W)$ and $\chi$ with the character through which it factors. This construction does not change $R^{T}$ nor $R_{\chi}^{T}$.

Assume that $T$ does not act stably on $X$. Then there must be a $\lambda \in Y(T)-$ $\{0\}$ such that $X$ does not contain a stable point for $\lambda$. Since $T$ acts now faithfully on $X$ we may assume that (after reindexing the $\alpha$ 's and possibly replacing $\lambda$ with $-\lambda)$ that there is a $d_{1}<d$ such that $\left\langle\lambda, \alpha_{i}\right\rangle=0$ if $1 \leq i \leq d_{1}$, and $\left\langle\lambda, \alpha_{i}\right\rangle>0$ if $d_{1}<i \leq d$. Let $W_{1} \subset W$ be the subspace spanned by $\left(w_{i}\right)_{1 \leq i \leq d_{1}}$ and define $T_{1}=T / \operatorname{im} \lambda$. Then the action of $T$ on $W_{1}$ factors through $T_{1}$.

For use below we define $R_{1}=k\left[W_{1}\right], X_{1}=\operatorname{Spec} R_{1}$. To solve

$$
\sum_{i} a_{i} \alpha_{i}=\chi
$$

with $a_{i} \geq 0$ is equivalent to solving

$$
\begin{gathered}
\sum_{i=d_{1}+1}^{d} a_{i}\left\langle\lambda, \alpha_{i}\right\rangle=\langle\lambda, \chi\rangle, \\
\sum_{i=1}^{d_{1}} a_{i} \alpha_{i}=\chi-\sum_{i=d_{1}+1}^{d} a_{i} \alpha_{i},
\end{gathered}
$$

with $a_{i} \geq 0$.

Hence let $\left(\chi_{j}\right)_{j=1, \ldots, N}$ be the characters of the form $\chi-\sum_{d_{1}+1}^{d} a_{i}^{(j)} \alpha_{i}$ where $\left(a_{i}^{(j)}=a_{i}\right)_{i=d_{1}+1, \ldots, d}$ is a solution of (20). It is clear that there are only a finite number of such solutions. Then $\left(\alpha_{i}\right)_{i=1, \ldots, d_{1}},\left(\chi_{j}\right)_{j=1, \ldots, N}$ factor through characters of $T_{1}$. We will use the same notations for these characters. A solution to (19) does now correspond to a solution of one of the equations $\sum_{i=1}^{d_{1}} a_{i} \alpha_{i}=\chi_{j}$. Hence $R_{\chi}^{T} \cong \bigoplus_{j=1}^{N} R_{1 \chi_{j}}^{T_{1}}$ and similarly $R^{T} \cong R_{1}^{T_{1}}$. It is easy to verify that these isomorphisms are compatible with each other.

By induction, it is now sufficient to show that for all $\lambda_{1} \in Y\left(T_{1}\right)-\{0\}$ and for all $j, R_{1 \alpha_{j} \circ \lambda_{1}}^{\lambda_{1}}$ is Cohen-Macaulay. Suppose that $R_{1 \chi_{j} \circ \lambda_{1}}^{\lambda_{1}}$ is not Cohen-Macaulay for some $\lambda_{1} \in Y\left(T_{1}\right)-\{0\}$. By the results in $\S 4.1$ we may assume that $X_{1}$ has a $\lambda_{1}$-stable point and $d_{\lambda_{1}}<d_{1}-1$ (possibly after replacing $\lambda_{1}$ with $-\lambda_{1}$ ). Let $\lambda_{1}^{\prime \prime} \in Y(T)$ be a lifting of $\lambda_{1}$ and let $\lambda_{1}^{\prime}=\lambda_{1}^{\prime \prime}-B \lambda$, where $B \in \mathbb{Z}$. Then $\lambda_{1}^{\prime}$ is also a lifting of $\lambda_{1}$. Furthermore if $B$ is large enough then $\left\langle\lambda_{1}^{\prime}, \alpha_{i}\right\rangle<0$ for $i=d_{1}+1, \ldots, d$. This implies that $T_{\lambda_{1}}^{c}=T_{\lambda_{1}^{\prime}}^{C}$ and $d_{\lambda_{1}}=d_{\lambda_{1}^{\prime}}$.

Since $R_{1 \chi_{j} \circ \lambda_{1}}^{\lambda_{1}}$ is not Cohen-Macaulay we know from Corollary 3.4.2 that there must be a solution

$$
b_{1}\left\langle\lambda_{1}, \alpha_{1}\right\rangle+\cdots+b_{d_{1}}\left\langle\lambda_{1}, \alpha_{d_{1}}\right\rangle=\left\langle\lambda_{1}, \chi_{j}\right\rangle
$$

where $\operatorname{supp}_{-}\left(b_{1}, \ldots, b_{d_{1}}\right)=T_{\lambda_{1}}^{c}$.

(22) is equivalent to

$$
b_{1}\left\langle\lambda_{1}^{\prime}, \alpha_{1}\right\rangle+\cdots+b_{d_{1}}\left\langle\lambda_{1}^{\prime}, \alpha_{d_{1}}\right\rangle+a_{d_{1}+1}^{(j)}\left\langle\lambda_{1}^{\prime}, \alpha_{d_{1}+1}\right\rangle+\cdots+a_{d}^{(j)}\left\langle\lambda_{1}^{\prime}, \alpha_{d}\right\rangle=\left\langle\lambda_{1}^{\prime}, \chi\right\rangle
$$

and $\operatorname{supp}_{-}\left(b_{1}, \ldots, b_{d_{1}}, a_{d_{1}+1}^{(j)}, \ldots, a_{d}^{(j)}\right)=T_{\lambda_{1}^{\prime}}^{c}$.

This, together with the fact that $d_{\lambda_{1}^{\prime}}=d_{\lambda_{1}}<d_{1}-1 \leq d-1$, implies that $R_{\chi \circ \lambda_{1}^{\prime}}^{\lambda_{1}^{\prime}}$ is not Cohen-Macaulay, which contradicts the hypothesis. 
Theorem 4.2.1 has no converse. In fact, in $\S 4.5$, we will give counterexamples to such a converse.

4.3 Stanley's criterion. In [7] Stanley presents a tractable condition on $\chi$ for $R_{\chi}^{T}$ to be Cohen-Macaulay. We now show how this criterion follows trivially from Corollary 3.4.2 and Proposition 3.4.5.

Theorem 4.3.1 [7]. Assume that $\chi=\sum_{i=1}^{d} u_{i} \alpha_{i}$ with $u_{i} \in$ ]-1,0]. Then $R_{\chi}^{T}$ is Cohen-Macaulay.

Proof. Assume that $R_{\chi}^{T}$ is not Cohen-Macaulay and $R_{\chi}^{T} \neq 0$ (otherwise the theorem is vacuous). Then according to Corollary 3.4.2 and Proposition 3.4.5 there exist $\lambda \in \Lambda \backslash\left\{\bar{\lambda}_{n}\right\}$ and $a=\left(a_{1}, \ldots, a_{d}\right) \in \mathbb{Z}^{d}$ such that

- $\chi=a_{1} \alpha_{1}+\cdots+a_{d} \alpha_{d}$,

- supp $_{-} a=\left\{i \mid\left\langle\lambda, \alpha_{i}\right\rangle \geq 0\right\}$.

We claim that we may assume that there is some $\alpha_{i}$ such that $\left\langle\lambda, \alpha_{i}\right\rangle>0$. Suppose that this is not the case. Then, since $\lambda \nsim \lambda_{n}$, the hyperplane defined by $\lambda$ intersects $\operatorname{pos}\left\{\left(\alpha_{i}\right)_{i=1, \ldots, d}\right\}$ in an $\alpha_{i}$ which is not an apex. Hence by displacing $\lambda$ slightly we may make $\left\langle\lambda, \alpha_{i}\right\rangle$ positive without changing $T_{\lambda}$. In this way we have not changed the equivalence class of $\lambda$.

Since by hypothesis $\left.\left.\chi=\sum_{i=1}^{d} u_{i} \alpha_{i}, u_{i} \in\right]-1,0\right]$ we obtain that

$$
\sum_{i=1}^{d}\left(a_{i}-u_{i}\right)\left\langle\lambda, \alpha_{i}\right\rangle=0 \text {. }
$$

For a particular $i$ there are two possibilities:

- $\left\langle\lambda, \alpha_{i}\right\rangle \geq 0$. Then $a_{i}<0$ and hence $a_{i}-u_{i}<0$.

- $\left\langle\lambda, \alpha_{i}\right\rangle<0$. Then $a_{i} \geq 0$ and hence $a_{i}-u_{i} \geq 0$.

It follows that for every $i$ either

$$
a_{i}=u_{i}=0 \quad\left(\text { and hence }\left\langle\lambda, \alpha_{i}\right\rangle<0\right)
$$

or

$$
\left\langle\lambda, \alpha_{i}\right\rangle=0 .
$$

Hence $\forall i:\left\langle\lambda, \alpha_{i}\right\rangle \leq 0$ which contradicts our hypothesis about $\lambda$.

4.4 Finiteness. In this section we will look for a condition under which there are only a finite number of $\chi$ such that $R_{\chi}^{T}$ is Cohen-Macaulay. To simplify things we will assume that $X$ has a stable point.

We start with two elementary lemmas, whose proof will be left to the reader.

Lemma 4.4.1. Let $E$ be a vector space, and assume that $x_{1}, \ldots, x_{m} \in E$. Let $B$ be the cone spanned by the $\left(x_{i}\right)_{i}$. Then there exists an $x$ such that

$$
(x+B) \cap\left(\sum_{i=1}^{m} \mathbb{Z} x_{i}\right) \subset \sum_{i=1}^{m} \mathbb{N} x_{i} .
$$

Lemma 4.4.2. Let $E$ be a vector space. Assume that $\left(B_{i}\right)_{i=1, \ldots, m}$ is a set of polyhedral cones in $E$ such that $E-\{0\}=\bigcup_{i=1}^{m}$ int $B_{i}$. Let $\left(u_{i}\right)_{i=1, \ldots, m} \in E$. Then $\bigcup_{i=1}^{m}\left(B_{i}+u_{i}\right)$ has bounded complement.

We will now verify the hypothesis for Lemma 4.4 .2 in the special case we are interested in. Denote by $\Lambda_{\max }$ the set of maximal elements for the partial ordering defined on $\Lambda-\{0\}$ by (14). 
Lemma 4.4.3. Assume that $X$ has a stable point. Then

$$
\bigcup_{\bar{\lambda} \in \Lambda_{\max }} \text { int } B_{\lambda}=E-\{0\} \text {. }
$$

Proof. Let $\chi \in E-\{0\}$. Write

$$
\chi=\sum_{i \in T} a_{i} \alpha_{i} \quad \text { all } a_{i}>0
$$

with $T$ minimal. It is then well known that the $\left(\alpha_{i}\right)_{i \in T}$ are independent (compare with [1, Example 2.3.6]). To see this assume that there is a nontrivial dependency $0=\sum_{i \in T} b_{i} \alpha_{i}$. Then for some $t, \chi=\sum_{i \in T}\left(a_{i}-t b_{i}\right) \alpha_{i}$ will have positive coefficients, but smaller support than $T$. This contradicts the choice of $T$. Hence there are $\mu \in E^{*}$ such that $\left\langle\mu, \alpha_{i}\right\rangle<0$ for all $i \in T$.

We then deduce from Lemma 3.6.5 that there are $\bar{\lambda} \in \Lambda_{\max }$ such that

$$
\chi \in \text { relint } \operatorname{pos}\left\{\left(\alpha_{i}\right)_{i \in T}\right\} \subset \text { relint } A_{\lambda} \subset \text { int } B_{\lambda},
$$

where the last inclusion follows from Lemma 3.5.2 and Lemma 3.6.6.

The following result will be the main theorem of this section.

Theorem 4.4.4. Assume that $X$ has a stable point. Then the following are equivalent.

1. $\forall \lambda \in \Lambda_{\max }:\left|T_{\lambda}\right|>u_{\lambda}$.

2 . There are only a finite number of $\chi \in X(T)$ such that $R_{\chi}^{T}$ is nonzero and Cohen-Macaulay.

Proof. (1) $\Rightarrow(2)$ Assume that (1) holds. Denote by $M$ the subsemigroup of $X(T)$ generated by $\alpha_{1}, \ldots, \alpha_{d}$. Since $X$ is assumed to have a stable point, it is easy to see that $M$ is a group. Furthermore it is clear that $R_{\chi}^{T} \neq 0 \Leftrightarrow \chi \in M$.

Let $\lambda$ be an arbitrary element of $\Lambda_{\max }$. Then by Theorem 3.6.3

$$
\tilde{H}^{i}\left(\Phi_{\lambda}, k\right)=\tilde{H}^{i-1}\left(\Phi_{\lambda}^{\prime}, k\right)= \begin{cases}k & \text { if } i=u_{\lambda}-1, \\ 0 & \text { otherwise }\end{cases}
$$

One deduces that if

$$
i=h-\left(\left|T_{\lambda}\right|-u_{\lambda}\right) \text {, then } \tilde{H}^{i+s-d_{\lambda}-1}\left(\Phi_{\lambda}, k\right) \neq 0 .
$$

Note that $h-\left(\left|T_{\lambda}\right|-u_{\lambda}\right)<h$ by hypothesis. Let $\chi \in M$ and assume that $R_{\chi}^{T}$ is Cohen-Macaulay. Then by (25) and Corollary 3.4.2 $\chi$ will not be of the form

$$
\sum_{i=1}^{d} a_{i} \alpha_{i} \text { where } \begin{cases}a_{i} \geq 0 & \text { if } i \in T_{\lambda}, \\ a_{i}<0 & \text { if } i \notin T_{\lambda}\end{cases}
$$

or equivalently $\chi$ will not be in the set

$$
\sum_{i \in T_{\lambda}^{c}}\left(-\alpha_{i}\right)+\sum_{i \in T_{\lambda}} \mathbb{N} \alpha_{i}+\sum_{i \in T_{\lambda}^{c}} \mathbb{N}\left(-\alpha_{i}\right) .
$$

Now by Lemma 4.4.1 there will be a $c_{\lambda} \in B_{\lambda}$ such that

$$
\sum_{i \in T_{\lambda}} \mathbb{N} \alpha_{i}+\sum_{i \in T_{\lambda}^{c}} \mathbb{N}\left(-\alpha_{i}\right) \supset\left(B_{\lambda}+c_{\lambda}\right) \cap M .
$$


Hence $\chi$ will not be an element of

$$
\bigcup_{\lambda \in \Lambda_{\max }}\left(B_{\lambda}+c_{\lambda}+\sum_{i \in T_{\lambda}^{c}}\left(-\alpha_{i}\right)\right) .
$$

Now from Lemma 4.4.1 and Lemma 4.4.2 it follows that (26) will have bounded complement. Hence there are only a finite number of possibilities for $\chi$.

(2) $\Rightarrow(1)$. Assume that there is a $\lambda \in \Lambda_{\max }$ such that $\left|T_{\lambda}\right|=u_{\lambda}$.

From the Hochster-Roberts theorem we know that $R^{T}$ is Cohen-Macaulay. Let

$$
\chi=\sum_{i \in T_{\lambda}} b_{i} \alpha_{i} \quad \text { all } b_{i} \in \mathbb{N} .
$$

Then to solve

$$
\chi=\sum_{i=1}^{d} a_{i} \alpha_{i} \quad \text { all } a_{i} \in \mathbb{N}
$$

we have to solve

$$
\sum_{i \notin T_{\lambda}} a_{i}\left(-\alpha_{i}\right)=\sum_{i \in T_{\lambda}}\left(a_{i}-b_{i}\right) \alpha_{i}
$$

Now the left-hand side of (28) is in $B_{\lambda} \cap E_{\lambda}=A_{\lambda}$ (using Lemma 3.6.6) and hence all $\left(a_{i}-b_{i}\right)_{i \in T_{\lambda}}$ must be positive (here we use the hypothesis that $\left.\left|T_{\lambda}\right|=u_{\lambda}\right)$. This means that there must be a one-one correspondence between the solutions to (27) and the solutions to

$$
0=\sum_{i=1}^{d} a_{i}^{\prime} \alpha_{i} \quad \text { all } a_{i}^{\prime} \in \mathbb{N}
$$

by putting $a_{i}=a_{i}^{\prime}+b_{i}$.

Therefore $R_{\chi}^{T} \cong R^{T}$ as $R^{T}$-modules and hence $R_{\chi}^{T}$ is Cohen-Macaulay. Since there are an infinite number of choices for $\chi$, we are done.

4.5 An explicit example. In this section we will determine, for a particular pair $(T, W)$, exactly when $R_{\chi}^{T}$ is Cohen-Macaulay. In particular we obtain counterexamples to the converses of Theorems 4.2.1 and 4.3.1. It should be noted that Stanley has already given a simple counterexample to the converse of Theorem 4.3.1 [8, Example 3.7]. If we analyze this example with the help of Corollary 3.4.2 then we see that it is based on the fact that for some $\lambda$ 's, $d_{\lambda}$ may be so large that the term in (10) involving $H_{X_{\lambda}}^{d_{\lambda}}\left(X, \mathscr{O}_{X}\right)$ does not contribute to $H_{X^{u}}^{i}\left(X, \mathscr{O}_{X}\right)$ for $0 \leq i<h$. This situation is somewhat special however, and it will not happen if $d$ is large enough and the weights of $W$ are distributed randomly in $X(T)$. In contrast our examples will not be based on the fact that the $d_{\lambda}$ may be large.

We will take $\operatorname{dim} T=2$. In that case $E \cong E^{*} \cong \mathbb{R}^{2}$ and the pairing $\langle$, $\rangle$ will be given by the usual inner product $\left\langle\left(x_{1}, x_{2}\right),\left(y_{1}, y_{2}\right)\right\rangle=x_{1} y_{1}+x_{2} y_{2}$. In the sequel we will denote the elements of $E$ and $E^{*}$ by the corresponding elements in $\mathbb{R}^{2}$.

We will choose $W=(-1,0)^{\oplus 2} \oplus(0,1)^{\oplus 2} \oplus(1,1)^{\oplus 2} \oplus(1,-1)^{\oplus 2}$. Here the exponent 2 was chosen to avoid the low-dimensional anomalies described above. Bigger exponents work equally well. 
TABLE 2

\begin{tabular}{|c|c|c|c|c|c|c|c|c|}
\cline { 2 - 9 } \multicolumn{1}{c|}{} & 1 & 2 & 3 & 4 & 5 & 6 & 7 & 8 \\
\hline$\lambda$ & $(2,1)$ & $(1,2)$ & $(-1,2)$ & $(-2,1)$ & $(-2,-1)$ & $(-1,-2)$ & $(1,-2)$ & $(2,-1)$ \\
\hline$d_{\lambda}$ & 6 & 6 & 6 & 4 & 2 & 4 & 2 & 4 \\
\hline$u_{\lambda}$ & 1 & 2 & 1 & 2 & 2 & 2 & 2 & 2 \\
\hline
\end{tabular}

Now $\Lambda-\{\overline{0}\}$ consists of eight elements, given by Table 2 .

In this table, $\lambda_{1}-\lambda_{8}$ are listed in counter clockwise direction and hence we deduce from Proposition 4.1.3 that $\Lambda_{\varnothing}=\left\{\bar{\lambda}_{1}, \bar{\lambda}_{3}, \bar{\lambda}_{6}\right\}$ and $\Lambda . .=\left\{\bar{\lambda}_{2}, \bar{\lambda}_{5}, \bar{\lambda}_{7}\right\}$. If we then apply Remark 4.1.2 we obtain Figure 1.

A few words of explanation are due here. The weights of $W$ have been represented by fat dots. Hence there are four fat dots, each representing a weight with multiplicity two. The origin has been marked by a +-sign.

The shaded area represents the elements $\chi$ of $X(T)$ where $R_{\chi}^{T}$ is not Cohen-Macaulay. Hence there are only a finite number of $\chi$ such that $R_{\chi}^{T}$ is Cohen-Macaulay. Since for every $\lambda \in \Lambda-\{\overline{0}\},\left|T_{\lambda}\right|>u_{\lambda}$, this is consistent with Theorem 4.4.4. Note that the $P O$-set structure defined by (15) on $\Lambda$ is
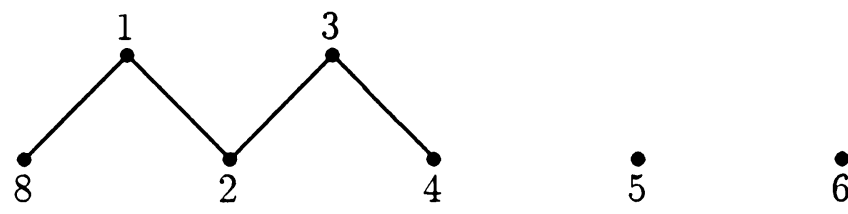

$\stackrel{\bullet}{6} \quad \dot{7}$

The interior of the region bounded by the dashed lines in Figure 1 are the characters of the form $\left.\left.\sum_{i=1}^{d} u_{i} \alpha_{i}, u_{i} \in\right]-1,0\right]$. In this case (but not always) these are precisely the characters which satisfy the hypothesis of

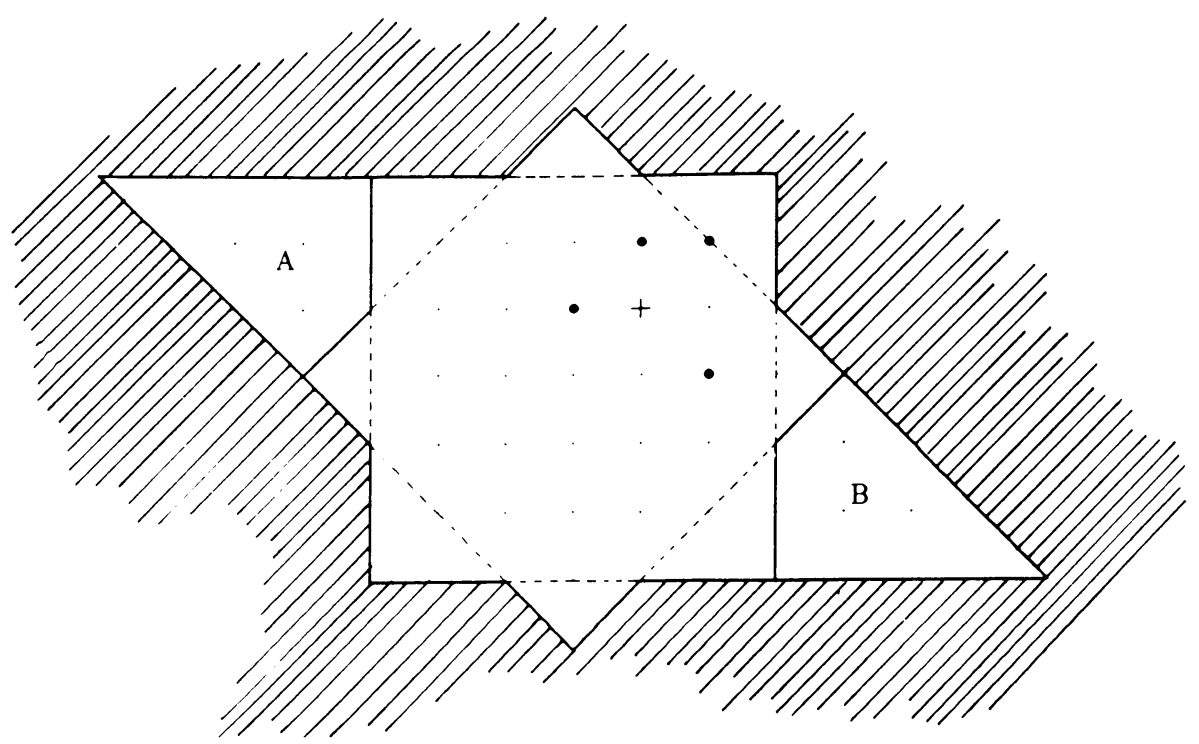

FIGURE 1 
Theorem 4.2.1. Since they do not fill all of the white area we obtain counterexamples to Theorems 4.2.1 and 4.3.1.

Furthermore the regions marked by $\mathrm{A}$ and $\mathrm{B}$ are those points where $H_{X_{\lambda_{4}}}^{d_{\lambda_{4}}}\left(X, \mathscr{O}_{X}\right)_{\chi}^{T}$ and $H_{X_{\lambda_{8}}}^{d_{\lambda_{8}}}\left(X, \mathscr{O}_{X}\right)_{\chi}^{T}$ are nonzero. But since $\lambda_{4}$ and $\lambda_{8}$ are contained in $\Lambda ., \lambda_{4}$ and $\lambda_{8}$ have no influence on the Cohen-Macaulayness of $R_{\chi}^{T}$.

4.6 The functional equation. Since $R_{\chi}^{T}$ is a $\mathbb{Z}^{d}$-graded object, it has a Poincaré series $P\left(R_{\chi}^{T}, t\right)$ where $t=\left(t_{1}, \ldots, t_{d}\right)$. It is well known that this Poincaré series is a rational function. Let $\psi=-\chi-\alpha_{1}-\cdots-\alpha_{d}$. In [7] and [8] Stanley shows that often the following functional equation holds

$$
P\left(R_{\chi}^{T}, t\right)=(-1)^{h} t_{1}^{-1} \cdots t_{d}^{-1} P\left(R_{\psi}^{T}, t^{-1}\right) .
$$

In [7] he also gives necessary and sufficient conditions for (29) to hold.

We will do the same thing (if $X$ has a stable point) using Corollary 3.4.2. As a result, we obtain that if $\operatorname{dim} T \leq 3$ and $X$ has a stable point then the fact that (29) holds, implies that $R_{\chi}^{T}$ is Cohen-Macaulay.

If $P\left(R_{\chi}^{T}, t\right), t=\left(t_{1}, \ldots, t_{d}\right)$ is a rational function then we will (as in [7]) denote by $P\left(R_{\chi}^{T}, t\right)_{\infty}$ the Laurent series expansion of $P\left(R_{\chi}^{T}, t\right)$ around $\infty$. This is then a Laurent series in $t_{1}^{-1}, \ldots, t_{d}^{-1}$. Furthermore the local cohomology modules $H_{\left(R^{T}\right)^{+}}\left(R_{\chi}^{T}\right)$ are Artinian and hence it is possible to define their Poincaré series as an element of $t^{\gamma} \mathbb{Z}\left[\left[t_{1}^{-1}, \ldots, t_{d}^{-1}\right]\right]$ for some $\gamma \in \mathbb{Z}^{d}$. There is the following identity [7],

$$
P\left(R_{\chi}^{T}, t\right)_{\infty}=\sum_{i=0}^{h}(-1)^{i} P\left(H_{\left(R^{T}\right)^{+}}^{i}\left(R_{\chi}^{T}\right), t\right) .
$$

Note however that $H_{\left(R^{T}\right)^{+}}^{i}\left(R_{\chi}^{T}\right)=0$ if $i<0$ or if $i>h$. Hence the bounds in the summation on the right-hand side of (30) are immaterial.

Using (10) we may then compute

$$
\begin{aligned}
P\left(R_{\chi}^{T}, t\right)_{\infty} & =\sum_{\lambda \in \Lambda} \sum_{i=-\infty}^{\infty}(-1)^{i} \operatorname{dim} \tilde{H}^{i+s-d_{\lambda}+1}\left(\Phi_{\lambda}, k\right) P\left(H_{X_{\lambda}}^{d_{\lambda}}\left(X, \mathscr{O}_{X}\right)_{\chi}^{T}, t\right) \\
& =\sum_{\lambda \in \Lambda}(-1)^{d_{\lambda}-s+1} \widetilde{\chi}\left(\Phi_{\lambda}\right) P\left(H_{X_{\lambda}}^{d_{\lambda}}\left(X, \mathscr{O}_{X}\right)_{\chi}^{T}, t\right) .
\end{aligned}
$$

Let us from now on assume that $X$ has a stable point. In that case $\Phi_{0}=\varnothing$.

We then obtain

$$
\begin{aligned}
P\left(R_{\chi}^{T}, t\right)_{\infty}= & (-1)^{d-s} P\left(H_{0}^{d}\left(X, \mathscr{O}_{X}\right)_{\chi}^{T}, t\right) \\
& +\sum_{\lambda \in \Lambda \backslash\{0\}}(-1)^{d_{\lambda}-s+1} \tilde{\chi}\left(\Phi_{\lambda}\right) P\left(H_{X_{\lambda}}^{d_{\lambda}}\left(X, \mathscr{O}_{X}\right)_{\chi}^{T}, t\right) .
\end{aligned}
$$

Since $X$ has a stable point, $d-s=h$. Furthermore using Corollary 3.3.2 we see that $P\left(H_{0}^{d}\left(X, \mathscr{O}_{X}\right)_{\chi}^{T}, t\right)=t_{1}^{-1} \cdots t_{d}^{-1} P\left(R_{\psi}^{T}, t^{-1}\right)$.

Hence we obtain

$$
\begin{aligned}
P\left(R_{\chi}^{T}, t\right)= & (-1)^{h} t_{1}^{-1} \cdots t_{d}^{-1} P\left(R_{\psi}^{T}, t^{-1}\right) \\
& +\sum_{\lambda \in \Lambda \backslash\{0\}}(-1)^{d_{\lambda}-s+1} \tilde{\chi}\left(\Phi_{\lambda}\right) P\left(H_{X_{\lambda}}^{d_{\lambda}}\left(X, \mathscr{O}_{X}\right)_{\chi}^{T}, t\right) .
\end{aligned}
$$


It also follows from Corollary 3.3.2 the $H_{X_{\lambda}}^{d_{\lambda}}\left(X, \mathscr{O}_{X}\right)_{\chi}^{T}$ all have distinct negative support.

We have now proved the following theorem.

Theorem 4.6.1. Assume that $X$ has a stable point. Then (29) holds if and only if for all $\lambda \in \Lambda \backslash\{0\}$ either $\tilde{\chi}\left(\Phi_{\lambda}\right)=0$ or there does not exist $a=\left(a_{1}, \ldots, a_{d}\right) \in \mathbb{Z}^{d}$ with supp $_{-} a=S_{\lambda}^{c}$ such that $\chi=a_{1} \alpha_{1}+\cdots+a_{d} \alpha_{d}$.

We may use this theorem to prove the following result.

Proposition 4.6.2. Assume that $X$ has a stable point and $\operatorname{dim} T \leq 3$. Then if (29) holds then $R_{\chi}^{T}$ is Cohen-Macaulay.

Proof. In this situation if $\lambda \neq 0$ then $\tilde{\chi}\left(\Phi_{\lambda}\right)=-\tilde{\chi}\left(\Phi_{\lambda}^{\prime}\right) \quad\left(\Phi_{\lambda}^{\prime}\right.$ was introduced in Proposition 3.4.4). $\Phi_{\lambda}^{\prime}$ is a subcomplex of the boundary complex of a spherical polytope of dimension $s-1$. It follows by inspection that if $\tilde{\chi}\left(\Phi_{\lambda}\right)=0$ then $\widetilde{H}^{\cdot}\left(\Phi_{\lambda}, k\right)=0$. Hence combining Corollary 3.4.2 and Theorem 4.6.1 yields that $R_{\chi}^{T}$ must be Cohen-Macaulay.

Remark 4.6.3. If $\operatorname{dim} T \geq 4$ then Proposition 4.6.2 is no longer true. For example if $\operatorname{dim} T=4$ then $\Phi_{\lambda}^{\prime}$ may be the disjoint union of a circle and a point. Then $\tilde{\chi}\left(\Phi_{\lambda}\right)=-\tilde{\chi}\left(\Phi_{\lambda}^{\prime}\right)=0$ but not all $\widetilde{H}^{i}\left(\Phi_{\lambda}, k\right)$ are zero.

It is possible to construct an explicit counterexample, but we will not bother to do so.

A result similar to Theorem 4.4 .4 can also be proved.

Theorem 4.6.4. Assume that $X$ has a stable point. Then there are only a finite number of $\chi$ such that (29) holds.

Proof. Given Theorem 4.6.1, the proof of the present theorem is completely similar to the proof of $1 \Rightarrow 2$ in Theorem 4.4 .4 except that (25) is replaced by $\tilde{\chi}\left(\Phi_{\lambda}\right) \neq 0$.

Note added in proof. A result, similar to Theorem 4.4.4, but valid for general reductive groups, meanwhile has been obtained by M. Brion (Sur les modules de covariants, preprint Institut Fourier 91). His proof is very different from ours.

\section{REFERENCES}

1. B. Grunbaum, Convex polytopes, Interscience, New York, 1976.

2. J. Herzog and E. Kunz, Der Canonische Modul eines Cohen-Macaulay-Rings, Lecture Notes in Math., vol. 238, Springer-Verlag, Berlin and New York, 1971.

3. M. Hochster and J. Roberts, Rings of invariants of reductive groups acting on regular rings are Cohen-Macaulay, Adv. in Math. 13 (1974), 313-373.

4. D. Quillen, Higher K-theories, Lecture Notes in Math., vol. 341, Springer-Verlag, Berlin and New York, 1973, pp. 85-139.

5. W. S. Massey, Singular homology theory, Graduate Texts in Math., Springer-Verlag, New York, 1980.

6. D. Mumford, Geometric invariant theory, Springer-Verlag, New York, 1982.

7. R. P. Stanley, Linear diophantine equations and local cohomology, Invent. Math. 68 (1982), 175-193.

8. _- Combinatorics and invariant theory, Proc. Sympos. Pure Math., vol. 34, Amer. Math. Soc., Providence, R.I., 1979. 
9. M. Van den Bergh, Trace rings of generic matrices are Cohen-Macaulay, J. Amer. Math. Soc. 2 (1990), 775-799.

10. __ Cohen-Macaulayness of modules of covariants (to appear).

Department of MAThematics, University of ANTWERP (UIA), WilRiJk, BelgiUm

Current address: Universite Louis Pasteur, UFR de Mathematique et Informatique, 7. Rue Rene Descartes, 67084 Strasbourg Cedex, France

E-mail address: vdbergh@math.u-strasbg.fr 